\title{
Ocular Adhesives: Design, Chemistry, Crosslinking Mechanisms, and Applications
}

Grissel Trujillo-de Santiago ${ }^{\mathrm{a}, \mathrm{b}, \mathrm{c}, \mathrm{d} \dagger}$, Roholah Sharifi ${ }^{\mathrm{a}, \mathrm{b} \dagger}$, Kan Yue ${ }^{\mathrm{a}, \mathrm{b}, \dagger}$, Ehsan Shrizaei Sani ${ }^{\mathrm{e}}$, Sara

Saheb Kashaf ${ }^{a, b}$, Mario Moisés Alvarez ${ }^{\text {a,b,c,d }}$, Jeroen Leijten, ${ }^{\text {a,b,f }}$, Ali Khademhosseini ${ }^{\text {a,b,e,g,h,i }}$, Reza

$$
\text { Dana }^{\mathrm{j}} \text {, Nasim Annabi }{ }^{\mathrm{a}, \mathrm{b}, \mathrm{e}, \mathrm{h}^{*}}
$$

aHarvard-Massachusetts Institute of Technology Division of Health Sciences and Technology, Massachusetts Institute of Technology, Cambridge 02139, MA, USA

bBiomaterials Innovation Research Center, Division of Biomedical Engineering, Department of Medicine, Brigham and Women's Hospital, Harvard Medical School, Boston 02139, MA, USA

'Microsystems Technologies Laboratories, MIT, Cambridge, 02139, MA, USA

${ }^{\text {d} C e n t r o ~ d e ~ B i o t e c n o l o g i ́ a-F E M S A ~ a n d ~ D e p a r t a m e n t o ~ d e ~ M e c a t r o ́ n i c a ~ y ~ E l e ́ c t r i c a, ~ E s c u e l a ~ d e ~ I n g e n i e r i ́ a ~ y ~}$

Ciencias, Tecnológico de Monterrey at Monterrey, CP 64849, Monterrey, Nuevo León, México

${ }^{e}$ Chemical and Biomolecular Engineering Department, University of California, Los Angeles, Los Angeles, CA 90095, USA

fDepartment of Developmental BioEngineering, MIRA Institute for Biomedical Technology and Technical Medicine, University of Twente, Enschede 7522, Netherlands.

gDepartment of Bioengineering, University of California - Los Angeles, Los Angeles, CA 90095, USA.

hCenter for Minimally Invasive Therapeutics (C-MIT), California NanoSystems Institute (CNSI), University of California - Los Angeles, Los Angeles, CA 90095, USA.

iDepartment of Radiology, David Geffen School of Medicine, University of California - Los Angeles, 10833 Le Conte Ave, Los Angeles, CA 90095, USA.

${ }^{j}$ Massachusetts Eye and Ear Infirmary and Schepens Eye Research Institute, Department of Ophthalmology, Harvard Medical School, Boston, MA 02114, USA.

${ }^{\dagger}$ These authors contributed equally to this work.

*Corresponding author 


\begin{abstract}
Closure of ocular wounds after an accident or surgery is typically performed by suturing, which is associated with numerous potential complications, including suture breakage, inflammation, secondary neovascularization, erosion to the surface and secondary infection, and astigmatism; for example, more than half of post-corneal transplant infections are due to suture related complications. Tissue adhesives provide promising substitutes for sutures in ophthalmic surgery. Ocular adhesives are not only intended to address the shortcomings of sutures, but also designed to be easy to use, and can potentially minimize post-operative complications. Herein, recent progress in the design, synthesis, and application of ocular adhesives, along with their advantages, limitations, and potential are discussed. This review covers two main classes of ocular adhesives: (1) synthetic adhesives based on cyanoacrylates, polyethylene glycol (PEG), and other synthetic polymers, and (2) adhesives based on naturally derived polymers, such as proteins and polysaccharides. In addition, different technologies to cover and protect ocular wounds such as contact bandage lenses, contact lenses coupled with novel technologies, and decellularized corneas are discussed. Continued advances in this area can help improve both patient satisfaction and clinical outcomes.
\end{abstract}

Keywords: Ocular, Bioadhesives and sealants, Natural and synthetic, Drug delivery

\title{
1. Introduction
}

Microsurgical suturing of ocular, in particular corneal, wounds is often associated with numerous drawbacks including post-operative astigmatism and requires a relatively high level of skill from the surgeon. Sutures can also provoke inflammation, lead to vascularization, and increase the risk 
of microbial infection, among other problems [1-5].

Ocular adhesives are promising alternatives to sutures. Sealants or adhesives have been used in ophthalmology for nearly five decades [6, 7]. These materials are typically polymers that are applied as fluids at the ocular wound site and are chemically or physically crosslinked to bind and hold tissues. Ocular adhesives not only prevent the patient and the surgeon from experiencing the drawbacks of sutures, but also can potentially offer important functionalities that are otherwise not easily attained. Some of these key added functionalities could be of great benefit to the patient, e.g. the feasibility to match the adhesive biomechanical properties to those of the native tissue, so the wound healing progresses without limiting tissue movement or affecting its function. Moreover, the adhesive material can be functionalized with pharmacological or biological compounds that prevent infection and inflammation and/or promote tissue regeneration.

The human eye architecture is highly complex; it possesses several layers of tissue precisely organized to allow the passage of light (Figure 1A). An ideal ocular adhesive should meet a number of characteristics depending on their specific application and the anatomical parts involved in the medical intervention (Figure 1B). Generally, it should be biocompatible, non-toxic, comfortable for the patient, easily and rapidly applied, adhesive to the ocular tissue, quickly seal the injured area, mimic the mechanical properties of the tissue, permeable to nutrients and gases, and offer a microbial barrier. Specific applications, such as corneal sealing, demand other characteristics such as transparency and a refractive index similar to the cornea. Additional desirable characteristics include cost-effectiveness, long storage stability, and the possibility of incorporating drugs or biological compounds [2, 8, 9].

Historically, commercial cyanoacrylate-based multi-purpose glues were the first adhesives used in ophthalmic practice, albeit used 'off-label' without FDA or other regulatory authority 
approval [10]. These adhesives offer fast and facile wound closure, but they are cytotoxic to eye tissues, possess a rough texture, require use of a 'bandage' contact lens and are very opaque precluding good vision when applied to the central cornea or examination of subjacent tissues by the examiner, thus not permitting evaluation of healing. Later, collagen-based adhesives appeared as a more biocompatible alternative to cyanoacrylate; however, they have low mechanical stability and adhesion strength. New strategies in biomaterials engineering have led to the formation of more advanced ocular adhesives [10]. These adhesives can be engineered from synthetic or natural polymers to match the physical properties of the native ocular tissue, and to tune their adhesion strengths. This design versatility allows for the development of effective ocular adhesives and sealants capable of closing wounds without impeding tissue movement and functions.

Herein, we review a spectrum of ocular adhesives ranging from synthetic materials (cyanoacrylates, PEG-based and dendrimers) to naturally derived polymers (proteins and polysaccharides). Fundamentals related to biomaterial design such as chemical nature, preparation methods, and physicochemical properties are discussed. In addition, the advantages and limitations of currently available ocular adhesives are summarized. We also discuss the concept of ocular adhesives as drug-delivery vehicles, and briefly review contact bandage lenses as an alternative to sealants in eye-wound-care. Finally, advances towards the development of functional decellularized corneas using biomaterials are described. 


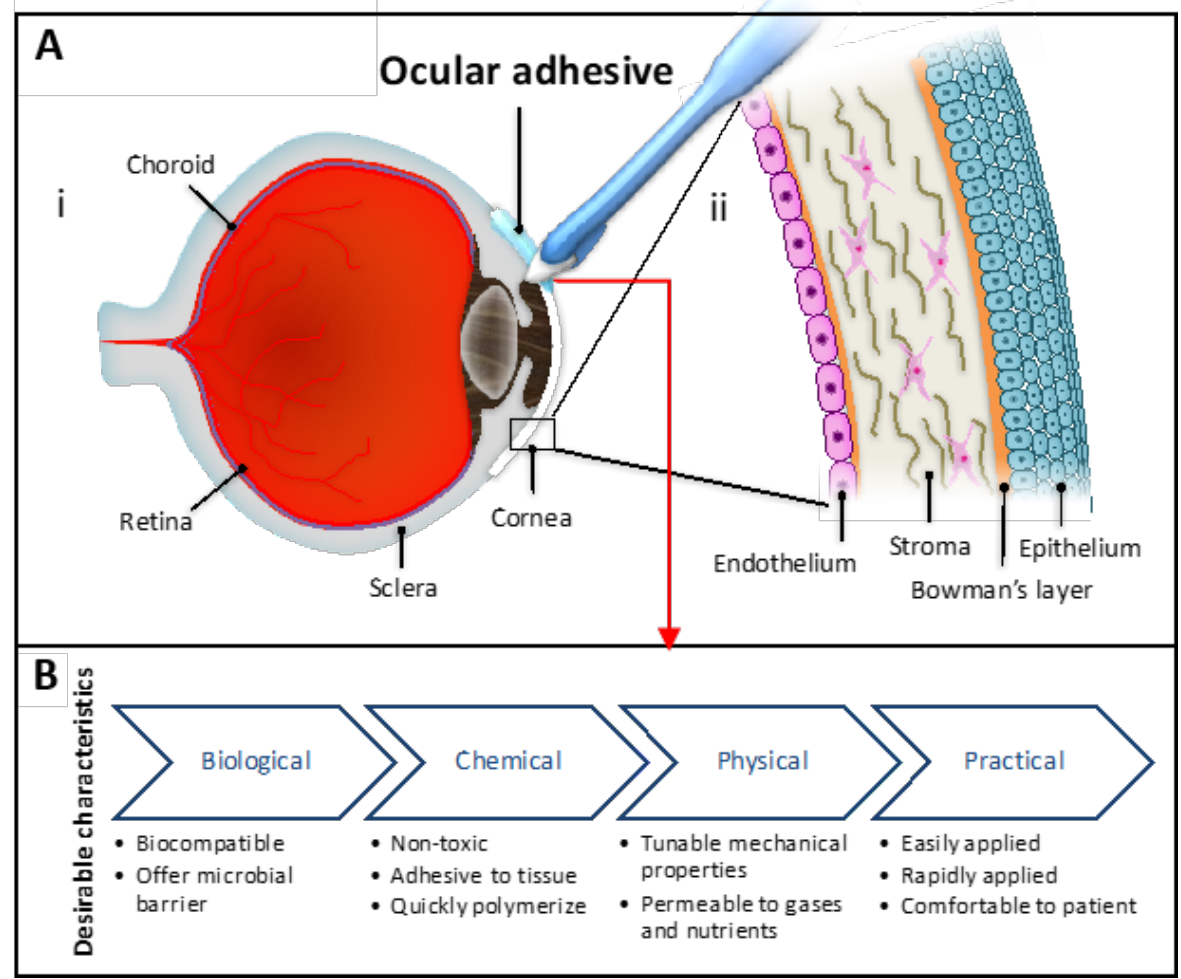

Figure 1. Desirable characteristics in an ocular adhesive and eye anatomy and cornea structure. (A) Anatomy of (i) eye and (ii) cornea. (B) Biological, chemical, physical, and practical characteristics that an ideal ocular adhesive should exhibit.

\section{Synthetic Polymer-based Tissue Adhesives}

Synthetic adhesives are materials often used in a wide spectrum of healthcare applications, including ophthalmology. These materials offer high tunability of a number of characteristics for specific ophthalmic applications such as chemical composition, mechanical properties, tissue adhesiveness, and degradation kinetics. Moreover, synthetic adhesives present added benefits with regard to ease of manufacturing, high purity, and low cost. The most widely explored synthetic adhesives used in ophthalmology are based on cyanoacrylates and linear polyethylene glycol (PEG) derivatives.

\subsection{Cyanoacrylate-based Ocular Adhesives}


Cyanoacrylate-based adhesives, also known as "superglues", are synthetic, multi-purpose tissue adhesives that have been used for a variety of general household usages as well as biomedical applications [11]. Bloomfield et al. were the first to report the ophthalmic in vivo use of methyl 2-cyanoacrylate in a rabbit model [12]. They applied the adhesive topically to the conjunctiva and the cornea, and sub-conjunctively to the sclera, which resulted in a tight wound closure in the first few postoperative days. Since this first report, the use of cyanoacrylates has become popular in ophthalmology. In ocular surgeries, cyanoacrylate-based adhesives are frequently used by ophthalmologists to seal eye wounds. Although they are not approved by Food and Drug Administration (FDA) for clinical ophthalmic use [13], these adhesives are used as an off-label for various applications such as cataract wound repair [14-21], leaking blebs [22-27], retinal detachment [28-31], scleral reinforcement [32], attachment of muscles to ocular prosthetics [33, 34], punctual occlusion [35, 36], blepharoplasty [37-39], temporary tarsorrhaphy [40-42], closure of corneal perforations [42], treatment of amblyopia [43], removal of deep corneal foreign bodies [44], stromal thinning repair [42], corneal descemetoceles [45], and exposure keratopathy [42, 46]. Although cyanoacrylate-based adhesives offer a quick, effective, and easy treatment of ocular wounds, they have several drawbacks including discomfort to the patient, cytotoxicity, and many other limitations that will be discussed in this section.

Cyanoacrylates are monomeric alkyl esters of $\alpha$-cyanoacrylic acid, which can be readily prepared via a condensation reaction between alkyl cyanoacetate and formaldehyde aided by a catalyst [47]. In general, cyanoacrylates have a high propensity to participate in polymerization reactions, which can proceed via an anionic or zwitterionic mechanism (Figure 2A). These reactions are initiated through a nucleophilic attack of alcohols, water, or amino acids present in the living tissues, and can later propagate to form alkyl methacrylate polymers [48]. The initiation reaction by amino 
acid residues of proteins is key for the strong binding of cyanoacrylate adhesives to the tissues [49]. Such strong binding, along with appropriate biomechanical properties and rapid polymerization (i.e. within 10-60 s), has made cyanoacrylates attractive for different medical applications.

However, the application of cyanoacrylate has been associated with irregular rough surfaces, heat generation, rapid polymerization, and cytotoxicity, limiting its usage for ophthalmic applications. Fast polymerization rate of cyanoacrylate is a characteristic that works both in favor and against this adhesive. While fast polymerization makes the process of sealing time-efficient and minimizes discomfort to the patient, it also complicates its application for the physician. For instance, in cornea perforation sealing, it is important to control the moisture of the tissue surface, and to rapidly apply a proper dose of cyanoacrylate so that a thin and even layer of the cyanoacrylate polymerizes in place to hold the tissue effectively [50]. Given these challenges, the selection of the proper technique for the application of cyanoacrylate-based adhesives in a particular intervention becomes critical for a successful clinical outcome. Rana et al. summarized different techniques to apply cyanoacrylate glues in corneal perforations [50]. The traditional technique consists of drying the ocular surface and gently adding a small drop of the cyanoacrylate glue on the affected tissue with a 20-gauge needle or other pointed instrument (Figure 2Bi). This method was successfully used for small perforations. For larger ocular wounds, they also used a modified method, which consisted of cutting a small non-adhesive bandage or surgical drape with a skin biopsy punch, holding this bandage with an infant cannula (without a needle) or with any small rod or applicator that can maintain adequate adhesion to the drape, adding a drop of glue on the bandage, and placing the bandage with the glue on the affected area (Figure 2Biii). Their results showed that the proper choice of the application techniques alone could result in improved 
surgery outcomes, even when using the same glue (Figure 2B); however, controlled application of cyanoacrylate glue to any surface, including the surface of the eye, remains a challenge

Cytotoxicity is another notable drawback of cyanoacrylates adhesives [51]. It is caused by the rapid degradation of the adhesives into cyanoacetate and formaldehyde, which can accumulate in tissues and induce acute and chronic inflammation [13, 52]. Various derivatives of cyanoacrylate esters such as n-butyl, $n$-heptyl, methoxypropyl, and octyl cyanoacrylates, have been developed to diminish cytotoxicity and lower the polymerization rate $[6,53]$. In general, cyanoacrylates with shorter alkyl chains show higher reactivity, faster degradation rate, and more serious toxic effects on tissues than their counterparts with longer alkyl chains [54]. Moreover, it has been reported that the tissue binding strengths of cyanoacrylate adhesives are inversely correlated with the lengths of the side chains (e.g., butyl cyanoacrylate had stronger binding than octyl cyanoacrylate) [55]. However, this is not a universally valid statement. Ricci et al. reported that octyl 2-cyanoacrylate achieved superior tensile strengths when bonding between the sclera and the muscle, as well as better adhesion at the edges of the wound, than cyanoacrylates with shorter alkyl chains such as methyl 2-cyanoacrylates. Cyanoacrylate adhesives have also shown some level of bacteriostatic and bactericidal effects [53]. Butyl 2-cyanoacrylate and ethyl 2cyanoacrylate exhibited bacteriostatic effects on Gram-negative and Gram-positive microorganisms in vitro and in vivo, especially when the adhesive was polymerized in situ [5658].

Cyanoacrylates can also be used in combination with other materials to improve their performance in particular interventions. For example, Lee et al. prepared a hybrid adhesive composed of pre-polymerized allyl 2-cyanoacrylate (PACA) mixed with poly L-lactic acid (PLLA) [59] to enhance the biocompatibility and mechanical properties of cyanoacrylates. Bona 
et al. reported using composite materials based on cyanoacrylates to enhance support and bonding strength in the context of strabismus surgery [60]. In this kind of surgery, there is need of a method to bond a sufficient area of sclera and extraocular muscles to avoid tissue slippage. For this purpose, the authors glued a polyglactin mesh with a butyl cyanoacrylate adhesive on the sclera of cadaveric eyes. Then, the mesh served as a scaffold to be attached to the muscle. The authors investigated the effects of polymerization time and mesh size (covered area) on bonding strength (the force needed to remove the mesh from the sclera). They demonstrated an improved adhesion with longer polymerization times and larger mesh areas (Figure 2C). This study provided a clinically relevant method to complement traditional protocols for bonding muscle to sclera in strabismus surgeries [60]. These studies suggest that the combination of cyanoacrylates with other materials can be used to engineer ophthalmic adhesives with enhanced performance.

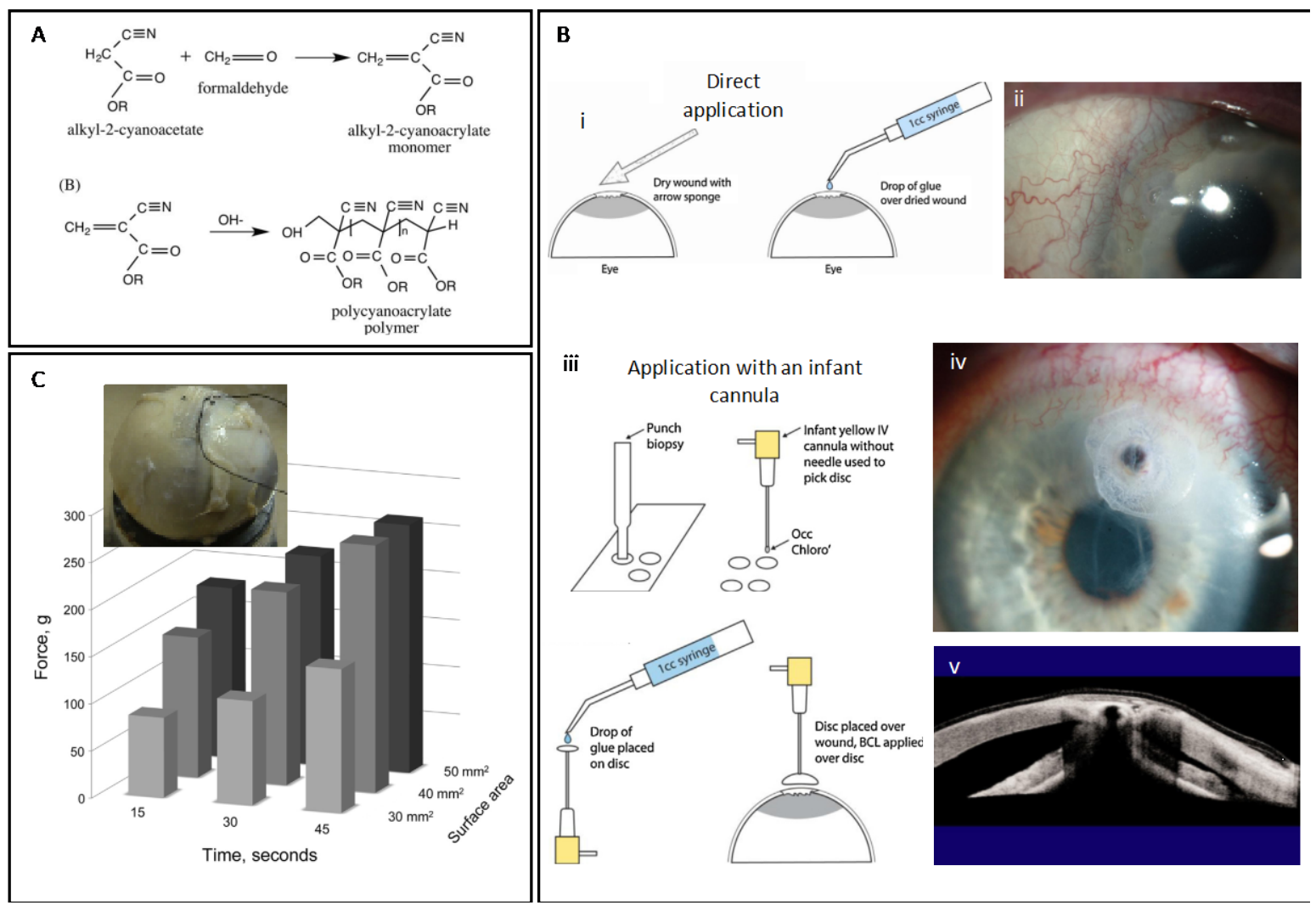

Figure 2. Structure, properties and clinical applications of cyanoacrylate adhesives. (A) Preparation and anionic 
polymerization reaction (crosslinking) of cyanoacrylate monomers. Adapted from Scognamiglio et al. [61] with permission from Wiley, copyright 2016. (B) Cyanoacrylate glue applications techniques, (i) traditional technique: direct application of a drop of cyanoacrylate glue on a pre-dried ocular tissue, and (ii) patient eye treated with this technique; (iii) infant cannula technique: a small disc of a non-adhesive material is cut with a punch biopsy, an infant cannula (without needle) is used to pick up and hold the disc, a drop of glue is applied on the disc, and finally the glue is placed gently on the pre-dried ocular tissue, (iv) patient eye treated with this technique, (v) corneal tissue repaired with this technique. Adapted from Rana et al. [50] with permission from Elsevier, copyright 2013. (C) A polyglactin mesh glued with cyanoacrylate on a cadaver eye for strabismus surgery application, and effect of the polymerization time and surface area on the bonding strength of the mesh to the eye tissue. Adapted from Bona et al. [60] with permission from Elsevier, copyright 2014.

In summary, despite their advantages (i.e. quick, easy, and effective treatment of ocular injuries), and their frequent off-label use in ophthalmic applications, the use of cyanoacrylates has been hampered by many shortcomings including infection [62], corneal endothelial polymerization [63], granulomatous keratitis [64], dacryocystitis [65], symblepharon formation [66], and unintentional entry into the anterior chamber [67]. In addition, the non-biodegradable nature of cyanoacrylates can lead to inflammatory responses, corneal neovascularization, foreignbody reactions, and tissue necrosis. Moreover, the application of cyanoacrylates in ophthalmic surgeries is limited due to the rigid, inflexible nature of the solidified adhesives, which is impermeable to fluid and metabolites, thus remaining as a foreign body. In addition, the nonbioabsorbable nature, lack of transparency, and rough surface limit healing of the underlying tissue, occlude vision, and cause discomfort when blinking $[4,5,53,68]$. Continued progress toward the modification of cyanoacrylates should alleviate some of the side-effects associated with their applications in ophthalmology. Moreover, engineering hybrid materials composed of 
cyanoacrylates and biocompatible polymers can be envisioned to afford better bioadhesives with enhanced biocompatibility than that of pure cyanoacrylate-based adhesives.

\subsection{PEG-based Adhesives}

PEG and its derivatives are frequently used for a wide range of biomedical purposes due to their remarkable advantages such as good solubility in both non-polar and polar solvents, antifouling properties, nontoxicity, and low immunogenicity [69]. In addition, the PEG backbone is amenable to a wide variety of chemical modifications, which makes it very versatile. For instance, PEG-based hydrogels have been functionalized with biochemical cues to tune their bioactivity, biodegradability, and mechanical properties, among others [70-72]. This versatility makes PEG an appealing material for ocular adhesives. In this section, we review PEG-based ocular glues, including those already in clinical use, and others currently under research and development.

ReSure ${ }^{\circledR}$ is an FDA-approved, PEG-based ocular adhesive. This product is specifically used to seal clear corneal incisions (CCI) commonly used in cataract surgery, and for the preclusion of fluid egress after cataract surgery [73]. ReSure ${ }^{\circledR}$ adhesive is based on two components: an Nhydroxysuccinimide (NHS)-terminated 4-arm PEG prepolymer and a tri-lysine amine crosslinker, which can yield stable hydrogels in less than $30 \mathrm{sec}$ after mixing. Clinical studies have shown several advantages of ReSure ${ }^{\circledR}$ over sutures such as improved comfort to the patient, faster healing, and better lubrication properties. In addition, ReSure ${ }^{\circledR}$ can sustain higher intraocular pressures (IOP; from 11-29 mmHg) [74], and it is more efficient than sutures in averting fluid egress in cataract surgery and in single-plane incisions (i.e. 4.1\% and 34.1\% fluid egress for PEG-based adhesive and suture groups, respectively) [73]. ReSure® has been also used in conjunction with 
flap lifting and scraping to prevent the recurrence of epithelial ingrowth [75] in laser-assisted in situ keratomileusis (LASIK) surgery. In addition, due to its synthetic origin, ReSure ${ }^{\circledR}$ cannot facilitate virus transmission, whereas other natural-based polymers might. However, ReSure ${ }^{\circledR}$ has some drawbacks and limitations. For example, the requirement for mixing of two components, and the need of rapid application after mixing (14-17 s) can be challenging in some circumstances. Also, ReSure ${ }^{\circledR}$ can only be used to seal incisions that are not actively leaking, and once applied it is stable for periods of no longer than 3 days [76]; additionally, it cannot fill in stromal defects (unlike cyanoacrylate) and can fall off easily when not covered by tissue [10, 76].

Alternative strategies have been reported to produce PEG-based hydrogel adhesives for ophthalmological use. These hydrogels are often produced by functionalizing the chain ends of linear PEG with acrylate, methacrylate, or other groups that are subject to free radical polymerization (e.g. poly(ethylene glycol) diacrylate (PEGDA)). Aided by a photoinitiator such as Eosin Y or Irgacure 2959, initiation of the free radical polymerization of the reactive groups on modified PEG derivatives can occur upon exposure to visible light [70] or ultra violet (UV) light [77], rendering 3D hydrogel networks (Figure 3Ai) [78].

The mechanical and biological performance of PEG-based hydrogels can be tuned by altering their physicochemical properties, such as polymer concentration, molecular weight of the PEG backbone, and crosslinking methods, among others. For instance, prepolymer concentration has been reported to have an important effect on mechanical properties, and therefore, the viability of encapsulated cells in the resulting hydrogel. Typically, increasing prepolymer concentration resulted in a higher compressive modulus, but may limit cell viability and proliferation in the 3D matrix [79]. The molecular weight of the PEG prepolymer can also affect the mechanical properties and swellability of PEG-based hydrogels. The wide-range tunability of the physical 
properties of PEG hydrogels enables the rational design and optimization of PEG-based adhesives in ophthalmology. For example, Yañez-Soto et al. [72] reported the development of PEGDA hydrogels for human corneal epithelial cell (HCEC) 2D cultures (Figure 3Aii). The authors produced hydrogels with particular topographic features by using a cast molding technique, and then immersed them into water until swelling equilibrium was reached. They found that the resolution of the topography was related to the molecular weight and concentration of the PEGDA used to form the hydrogel. In order to obtain high-resolution features, the ratio between the hydrogel volume before and after equilibrium swelling had to be low, which was achieved by lowering the PEGDA molecular weight, and polymer concentrations [72] (Figure 3Aii). Interestingly, the molecular weight also had an effect on non-specific binding of HCECs. Flat hydrogels fabricated with low molecular weight PEGDA (700 g/mol) rendered a higher degree of nonspecific cell binding than that of higher molecular weight PEGDA (3400 g/mol; Figure 3Aiii).

PEG-based hydrogels are known to inhibit non-specific binding of cells. However, enhanced cellular interaction with PEG-based hydrogels can be achieved via chemical modification to introduce peptide cell binding motifs. For example, PEGDA hydrogels can be modified by a Michael addition of cysteine-bearing arginylglycylaspartic acid (RGD) peptides (Figure 3Bi) to enable specific RGD-integrin attachment of HCECs to the PEGDA hydrogel surface (Figures 3Bii and 3Biii). This flexibility of chemical modification affords a diverse range of functions of PEGbased adhesives [80].

Hoshi et al. used a porcine ex vivo model in which the retina was detached after the vitreous gel was removed from the eyecups, and a PEG-based adhesive (FocalSeal ${ }^{\circledR}$ from Genzyme Corporation) was applied to cover the tear. Upon exposure to xenon light (450-500 nm) for 40-60 sec, the prepolymer solution formed a solid, crosslinked hydrogel. The adhesive remained attached 
for $24 \mathrm{~h}$ despite a forceful squirt of balanced salt solution, showed little inflammatory reaction in the eyes during 28 days of follow up, and the electroretinography also did not show any abnormality or inflammation [81]. In addition, the authors also conducted an in vivo study to investigate the application of this photo-curable PEG-based sealant for the closure of sutureless sclerotomies (surgical incision of the sclera) in microincisional vitrectomy surgery (MIVS) using a Dutch pigmented rabbit eye model (Figure 3Ci). They showed that 23-gauge vitrectomy incisions in rabbit eyes could be successfully closed by this photocured, PEG-based adhesive (Figure 3Cii) and that the sealant did not cause immoderate inflammation as observed by histological analysis (Figure 3Ciii).
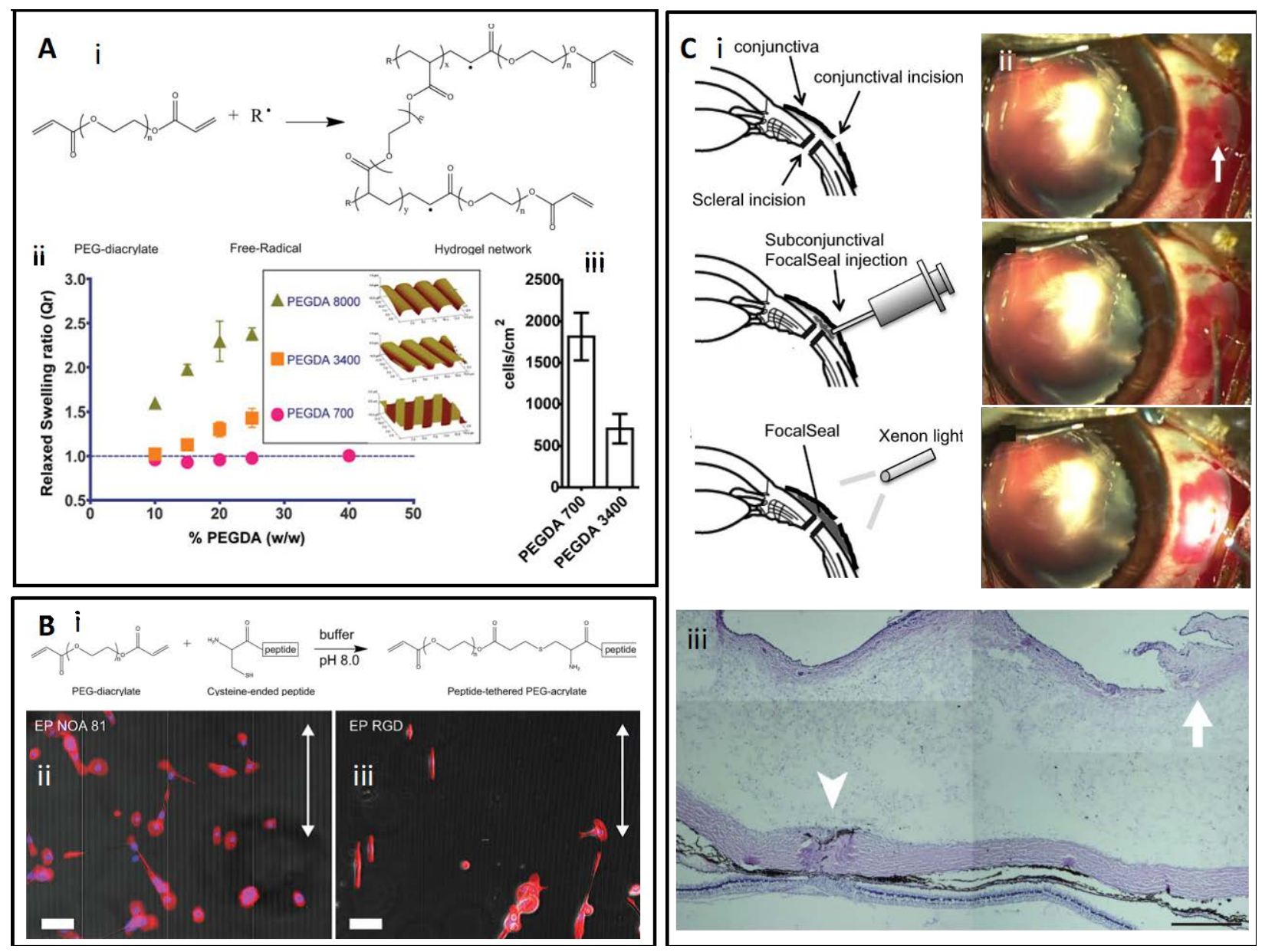

Figure 3. Molecular structure and properties of PEG-based adhesives. (A) PEGDA synthesis (i) PEG functionalized with diacrylated groups formed a 3D hydrogel network in the presence of a photoinitiator upon 
exposure to light. Physical and biochemical properties of resulting hydrogels were customized by modifying hydrogel formulations. (ii) Effect of PEGDA molecular weight on water swelling behavior and on pattern resolution on molded PEGDA hydrogels. (iii) Effect of PEGDA molecular weight on non-specific cell attachment. Adapted from YanezSoto et al. [72] with permission from Wiley, copyright 2013. (B) (i) Chemical modifications to functionalize PEGDA hydrogels with RGD peptide motifs, Micro-molded PEGDA hydrogels (ii) with and (iii) without RGD functionalization to tune cell attachment and to direct the alignment of human coronal epithelial cells. Scale bar: 100 $\mu \mathrm{m}$. Adapted from Yanez-Soto et al. [72] with permission from Wiley, copyright 2013. (C) (i) Scheme of the sealing of scleral incisions using PEG adhesive, (ii) Photographic images of sealing of a scleral incision in a rabbit vitrectomy model with PEG adhesive, (iii) Photographs of sectioned samples of a sclerotomy site closed with the PEG-based adhesive (the conjunctival incision is indicated by the arrow). Excessive inflammation is not evident. (Scale bar: 500 $\mu \mathrm{m})$. Adapted from Hoshi et al. [81] with permission from Association for Research in Vision and Ophthalmology, copyright 2016.

PEG terminals can be also functionalized with nucleophilic groups, which will subsequently react with electrophilic groups of a second component. However, the selection of the proper nucleophile-electrophile chemistry that can take place quickly at the body temperature under physiological conditions, without producing toxic by-products, has proven to be challenging. In addition, such reactions require a high degree of chemoselectivity between the designed nucleophile-electrophile pairs, due to the presence of a wide range of other functional groups such as amines, thiols, hydroxyls, and carboxylates in native tissue environments, which may interfere with the crosslinking reaction. For example, PEG functionalized with amines and thiols can react with complementary $N$-hydroxysuccinimide esters to form amide or thiol ester crosslinked hydrogels, respectively. Depending on the molecular weights of PEGs and the prepolymer concentrations, hydrogels could form in 5 s to 5 min [82]. Linear PEGs functionalized with succinimidyl succinate (SS) or succinimidyl propionate (SPA) groups could form crosslinked networks with a complementary tetra-functional amine-terminated PEG, which could tolerate higher load before failure than fibrin-based adhesives or photocrosslinkable acrylic adhesives. 
Even though the hydrogels made from PEG-SPA have superior mechanical properties and stability (up to several months), histological evaluation revealed that these adhesives can cause severe damage when applied to rabbit's eyes [83]. Moreover, hydrogels fabricated from PEG functionalized with succinimidyl glutarate (SG) and thiol groups showed superior mechanical properties compared with the PEG-SPA and PEG-SS based adhesives [83]. Kalayci et al. reported that the NHS-terminated 4-arm succinimidyl-glycolate PEG and complementary aminefunctionalized 4-arm PEG could form crosslinked hydrogels in seconds, which were stable for up to six weeks. Application of this hydrogel to central corneal incisions that were 1-5 $\mathrm{mm}$ in size in successfully sealed the incision in an ex vivo rabbit model. Also, the adhesive-treated eyes could tolerate higher leaking pressure in all wound models compared with a suture-treated eye [84], thus suggesting the potential of these adhesives for successful eye wound closure.

The use of PEG-based materials has also been extended to develop artificial corneas [85]. For example, the interpenetrating network of PEG functionalized with poly(acrylic acid) exhibited desirable mechanical properties, transmissibility and diffusion coefficients (i.e. Young's modulus of $2 \mathrm{MPa}$ for $20 \%$ (w/w) concentration, 96\% light transmissibility and ideal diffusion coefficients for oxygen and nutrients). In vivo application of this hybrid adhesive showed long-term stability and sufficient biocompatibility in a rabbit model [85].

\subsection{Dendrimer-based Adhesives}

Dendrimers are polymeric repetitive hyper-branched macromolecules that have gained attention in the scientific community because of their particular physical and chemical properties such as multifunctionality, monodispersed molecular weights, and high surface area to volume ratio [86-89]. Such properties, along with their highly tunable chemical structures, make them suitable for various biomedical applications [90] including the development of sealants and 
bioadhesives [91]. A dendrimer can be synthetized by the chemical condensation of successive layers of monomers from a central moiety (divergent synthesis), or by the synthesis of multiple branches to later fuse them to a central core (convergent synthesis). In any case, the result is a highly symmetric and hyper-branched macromolecule that is structurally composed of three main zones: the central core, the internal branching layers, and the peripheral moieties [90, 92, 93].

Dendrimers can be synthesized from natural metabolic compounds including amino acids such as lysine, valine, and leucine [93], sugars [94], $\alpha$-hydroxy acids, fatty acids [92], and metabolic intermediates, such as citric acid, fumaric acid, pyruvic acid, and succinic acid [95, 96]. Along with these small-molecule monomers, modified macromers based on PEG, polycaprolactone (PCL), and polytrimethylene carbonate (TMC), among others, offer additional flexibility in terms of monomer selection and the ability to match dendrimer structures to specific functional needs [2].

In a dendrimer, each layer is called a generation $(G)$. As the number of generations $\left(G_{n}\right)$ increases, the dendrimer becomes more branched (e.g. $\mathrm{G}_{0}$ vs. $\left.\mathrm{G}_{4}\right)$ (Figure 4A) and much larger. Dendrimers can be crosslinked to form 3D hydrogel networks. Crosslinking methods for dendrimers are very similar to those described for PEG-based adhesives: (i) chemical modification with acrylate groups to further photopolymerize the polymer in a presence of a photoinitiator, and (ii) modification with nucleophilic groups that will react with electrophilic groups of a second polymer [2]. Dendritic molecules can be used as the bulk polymers to provide adhesiveness to hold the tissue, create a protective barrier to cover the ocular wound, or as the crosslinker for another polymer that constitute the main network (e.g. PEG and collagen) [97].

Grinstaff et al. [2] synthesized a series of first- to fourth-generation $\left(\mathrm{G}_{1}-\mathrm{G}_{4}\right)$ photocrosslinkable hybrid dendrimers to seal corneal lacerations. These dendrimers, termed ([Gn]- 
PGLSA)2-PEG, were synthesized from PEG, glycerol, and succinic acid units [91]. A method to seal a linear incision in an ocular globe using these photocrosslinkable dendritic adhesives is shown in Figure 4B. First the incision is made in the ocular tissue, a precise amount of the dendrimer-based sealant is applied on the pre-dried wound, and finally photo-crosslinked using a laser beam (argon ion; $200 \mathrm{~mW} ; 1 \mathrm{sec)}$ [98]. Recent studies reported different generations of dendritic copolymers of PEG, glycerol, and succinic acid [([Gn]-PGLSA-MA $\left.)_{2}-P E G, n=0-4\right]$ as candidates to repair corneal lacerations [78, 99]. Both the chemical nature and the overall architecture of the dendrimer (i.e. degree of branching, number of generations) can determine the functionality of the ocular adhesive. For instance, Grinstaff found that the $\left[\left(\left[\mathrm{G}_{0}\right]-\mathrm{PGLSA}-\mathrm{MA}\right)_{2}\right.$ PEG] copolymer was not able to effectively crosslink to afford leak-tight sealing, and larger generations could be easily peeled off without providing a sufficient sealing [99]. Overall, ([G $\left.\mathrm{G}_{1}\right]$ PGLSA-MA)2-PEG exhibited the best performance in ocular globes with a linear incision [99]. The recorded leaking pressure from this adhesive $(109.6 \pm 82.7 \mathrm{mmHg})$ exceeded that of a traditional suture treatment $(75 \pm 27.8 \mathrm{mmHg})$. Moreover, this dendrimer-based adhesive showed adequate efficiency to repair corneal perforations of $4.1 \mathrm{~mm}$, to seal the flap originated in a LASIK operation, and to secure a transplant of cornea ex vivo [98-100].

An in vivo chicken model was used to test ([G $\left.\mathrm{G}_{1}\right]$-PGLSA-MA) $)_{2}$-PEG for corneal wound healing [2]. Twenty $\mu \mathrm{L}$ of the dendritic adhesive was applied on $4.1 \mathrm{~mm}$ full-thickness linear corneal wounds and subsequently crosslinked upon exposure to a laser, resulting in successful sealing of $97 \%$ of the laceration. While the adhesive fully degraded by day 14 , the lacerations sealed with the dendritic formulation showed a more uniform histology compared with the corneal wounds treated with sutures. The histological cross-sections of the tissue repaired using [ $\left.\mathrm{G}_{1}\right]-$ PGLSA-MA) 2 -PEG dendritic adhesive after 28 days showed that the dendritic adhesives led to a 
smoother stromal layer, with no discontinuities of Bowman's layer and no detachment of the stromal and epithelial layer, compared to tissue repaired with sutures (Figure 4C). However, the adhesive-treated group expressed wider corneal scars in contrast with the suture treated group, which could influence visual acuteness. This may originate from an insufficient cohesiveness of the adhesive to keep the edges of the wound closed [2]. In addition, this particular dendrimer-based adhesive required argon-laser radiation to induce effective crosslinking between dendrimers and cornea, which may add cost and complexity to the surgical procedure and could lead to possible side effects. 

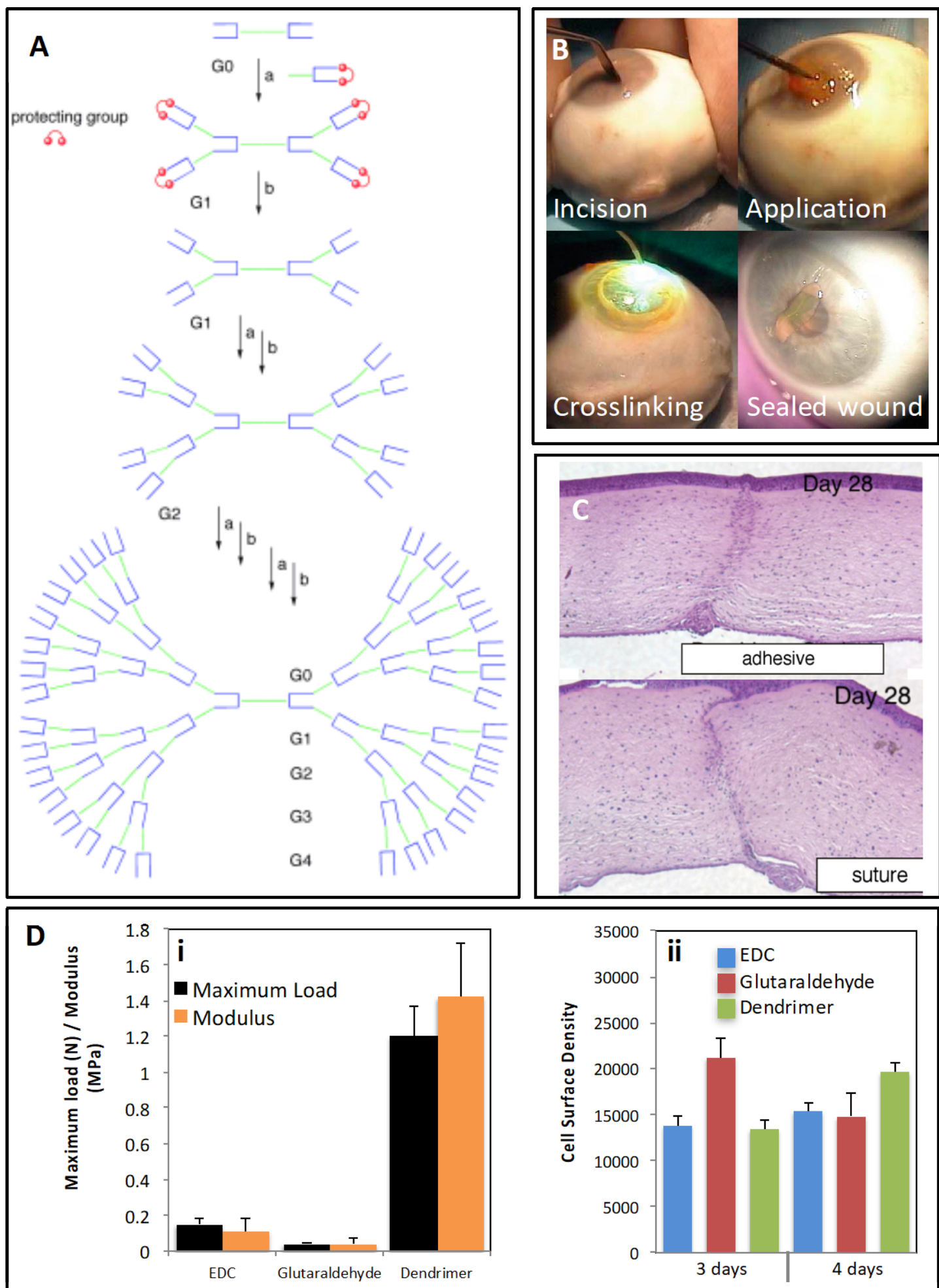

Figure 4. Structure, properties, and applications of dendritic adhesives. (A) Schematic illustration of different generations of dendritic adhesives from $\mathrm{G}_{0}$ to $\mathrm{G}_{4}$, which were prepared via divergent synthesis. The central core, inner branches, and peripheral groups were three main structural regions of these macromolecules. Adapted from Grinstaff et al. [99] with permission from Wiley, copyright 2002. (B) A method to apply and crosslink a ([G $]$-PGLSAMA)2- 
PEG dendritic adhesive. An incision of $4.1 \mathrm{~mm}$ was made on enucleated an eye cornea, 15-20 $\mu \mathrm{L}$ of adhesive was placed on the surface of the pre-dried wound, the adhesive was photo-crosslinked using argon laser (diffuse beam; $200 \mathrm{~mW}$; $1 \mathrm{sec}$ ), the sealed wound withstood an IOP of $109.6 \pm 82.7 \mathrm{mmHg}$ before leakage. Adapted from Velazquez et al. [98] with permission from American Medical Association, copyright 2004. (C) Images of histology transversal cuts of chicken cornea wounds treated with a ([G $\left.\mathrm{G}_{1}\right]$-PGLSAMA)2-PEG dendritic adhesive and suture after 28 days. Adapted from Grinstaff et al. [2] with permission from Elsevier, copyright 2007. (D) Dendrimers used as crosslinking agents of collagen hydrogels. (i) Mechanical properties and (ii) cell growth of collagen hydrogel crosslinked with generation 2 polypropyleneimine octaamine dendrimer compared to collagen hydrogels crosslinked with 1-ethyl-3(3-dimethyl aminopropyl) carbodiimide hydrochloride and glutaraldehyde. Adapted from Duan and Sheardown et al. [97] with permission from Elsevier, copyright 2006.

Similar to linear PEG-based adhesives, dendritic macromolecules can also be functionalized with nucleophilic groups, which subsequently react with another counterpart that has electrophilic moieties. For example, the rapid formation of a thiazolidine bond between the macromolecules with the aldehyde terminal groups and a counterpart with the 1,2-aminothiol moieties in cysteine residues at room temperature formed crosslinked hydrogel networks that were stable for $\mathrm{pH}$ values ranging from 3 to 9 [91]. In vitro studies showed that 5 min after applying PEG-dendritic adhesive (([G $\left.\left.\left.]_{1}-P G L S A-M A\right)_{2}-P E G\right)\right)$ to a 3-mm corneal linear incision, the sealant tolerated higher pressures applied to the wound (up to $184 \pm 79 \mathrm{mmHg}$ ) compared to the conventional sutures (up to $54 \pm 16 \mathrm{mmHg}$ ) or self-sealed wounds (up to $24 \pm 8 \mathrm{mmHg}$ ) [98]. This adhesive was also used to seal LASIK flaps [100] and repair corneal lacerations [78].

In order to improve the long-term stability of dendritic bioadhesives, Wathier et al. utilized another peptide ligation reaction, a pseudoproline formation, where a dendron with $N$-terminal cysteine moieties was reacted with PEGs with terminal aldehyde-ester moieties to create highly stable hydrogels [101]. Such hydrogels were optically transparent and showed a very slow rate of degradation, with only $10 \%$ weight loss in 6 months in a humidity chamber. The mechanical properties of this hydrogel were found to be strongly dependent on prepolymer concentrations. The compressive moduli for hydrogels made from 20, 33, or 50\% (w/w) prepolymer solutions were 200, 
570, and $850 \mathrm{kPa}$, respectively. In vitro application of the hydrogel into 8-mm trephined central corneal laceration was shown to lower the number of the sutures required to secure the autograft and increased the leaking pressure from $13 \pm 5 \mathrm{mmHg}$ (sutured group) to $63 \pm 7 \mathrm{mmHg}$ (hydrogel applied to sutured wound). However, the application of adhesives did not secure the autograft, indicating that the hydrogel might lack sufficient adhesiveness and coherence.

Oelker et al. engineered aldehyde-terminated PEG precursors, including PEG-butyraldehyde (B-Ald PEG), PEG-2-oxoethyl succinate (E-Ald PEG), and PEG-propionaldehyde (P-Ald PEG) to increase the stability of dendrimer PEG-based adhesives [1, 102]. The crosslinking reaction occurred between amine functionalities of the cysteine moieties of Lys3 Cys4 dendron with P-Ald or B-Ald PEG, forming thiazolidine bond, that were prone to hydrolysis. On the other hand, the reaction between the Lys3Cys4 dendron and E-Ald PEG formed thiazolidine bonds which could rearrange through $\mathrm{O}, \mathrm{N}$-acyl migration and generate more stable pseudoproline bonds. These hydrogels could seal full thickness central corneal lacerations of $4.1 \mathrm{~mm}$ in an ex vivo model to tolerate IOPs comparable to those treated with a single suture [102].

Dendrimers have also been used as crosslinker agents to form adhesive hydrogels for ocular applications. Duan et al. developed collagen-based scaffolds crosslinked with a dendrimer for cornea tissue engineering [97]. They used a collagen solution and a G-2 poly-propyleneimine octaamine dendrimer to produce a highly crosslinked collagen-based network. Compared to other crosslinking agents (i.e., glutaraldehyde and 1-ethyl-3-(3-dimethyl aminopropyl) carbodiimide hydrochloride, EDC), the crosslinked dendrimer scaffolds exhibited superior mechanical performance (Figure 4Di) and supported HCEC proliferation on their surface (Figure 4Dii).

Dendrimer-based adhesives hold high promise as ophthalmic sealants. Conceivably, these hyperbranched compounds can be tailored and used for broad spectrum of applications. They can be also 
engineered for the controlled release of different compounds [90] (e.g. antibiotics, growth factors, and chemical messengers) to promote tissue healing. The complexity and high cost of synthesis of dendrimers may be the most significant obstacle that prevents their applications in a clinical setting. Besides the high cost to synthesize such complex dendritic materials, manipulation of these adhesives into injured eye tissue can also be challenging during the surgery, as the mixing of two components may lead to fast formation of covalent networks in a short period.

\section{Naturally derived Polymer-based Adhesive}

\subsection{Protein-Based Adhesives}

The use of protein-based materials to repair ocular tissues has recently gained significant attention. So far, several natural proteins such as fibrin, collagen, gelatin, and silk [103], have been studied with the aim of repairing or sealing ocular wounds. Some of these materials have received FDA approval for use as tissue adhesives (but not specifically for ophthalmological use), and some, mainly fibrin-derived products, have become well-established commercial products. Examples are CryoSeal ${ }^{\circledR}$ (Thermogenesis, CA, USA), Tisseel and Artiss (Baxter, CA, USA), Evicel ${ }^{\circledR}$ (Johnson \& Johnson, NJ, USA), and BioGlue ${ }^{\circledR}$ (Kennesaw, GA, USA) [10]. VitaGel (Orthovita, PA, USA), a system to prepare fibrin glue from the blood of the patient, was recently approved and launched. Many other protein-based sealants are still at the research and development stage.

\subsubsection{Fibrin and Fibrinogen-Based Adhesives}

Fibrin sealants, or fibrin glues, together with cyanoacrylate-based adhesives, remain the most commonly used suture substitutes in ophthalmology. The rationale behind the use of fibrin as sealants is to mimic the biological process of fibrin clot formation. Fibrin (from the Latin word fibra, fiber) is an elastic and filamentous protein produced from fibrinogen catalyzed by the enzyme 
thrombin. This reaction naturally takes place in the last stage of the coagulation cascade, forming an interacting fibrous network or blood clot. Thrombin catalyzes the conversion of fibrinogen to fibrin by cleavage of the peptides A and B in the central fibrinogen nodule in the presence of calcium ions (Figure 5Ai) [104, 105]. The release of fibrin peptide triggers protofibril formation. A series of electrostatic and conformational changes follows, enabling polymerization of fibrin molecules into long and highly branched fibers, which then aggregate laterally to form the final shape of the fibrin [105]. The structure and function of fibrin and fibrinogen [106-109], and the process of clot formation have been described in detail in literature (Figure 5Aii) [110].

In general, fibrin glues are complex formulations derived from mammalian plasma (mainly human and bovine) that combine fibrinogen (typically derived from human plasma) with thrombin (bovine or recombinant) to form fibrin clots. Fibrin clots, formed by adding different concentrations of thrombin solutions to purified fibrinogen, develop different crosslinking densities as observed by SEM (Figure 5Aiii). In vivo studies on fibrin adhesives have confirmed their biocompatibility, biodegradability, and re-absorbability $[105,111]$. The adhesive strengths of fibrin sealants, as measured by the lap shear method, range from $1 \mathrm{kPa}$ to $27.9 \mathrm{kPa}$ as a function of fibrin concentration (Figure 5Aiv) and gelation time. For example, a $7.2 \mathrm{mg} / \mathrm{mL}$ fibrin glue rendered a clot with an adhesive resistance of $1 \mathrm{kPa}$ after gelation for $5 \mathrm{~min}$, whereas a $34.5 \mathrm{mg} / \mathrm{mL}$ fibrin glue showed an enhanced resistance of $27.9 \mathrm{kPa}$ at a gelation time of $90 \mathrm{~min}$ [112]. In general, the adhesive strengths of fibrin glues are lower than other crosslinked protein-based biomaterials (Figure 5Aiv) but are sufficient for a vast range of ocular applications.

The use of fibrin to repair corneal injuries was first reported in the early 2000s. Today, its application has been well documented to solve many ophthalmic surgical problems. For instance, Sharma et al. demonstrated that fibrin glues can be effective in healing some corneal perforations 
[113]; however fibrin based glues are notoriously poor in adhering to wet surfaces. Fibrin glues also presented several advantages in terms of accelerated speed of healing (1 week faster) and less corneal vascularization when compared to cyanoacrylates glues. However, compared to cyanoacrylates, fibrin glues required a longer time to gel after application (2 or $3 \mathrm{~min}$ ) [113]. Similarly, Bahar et al. used a fibrin-based adhesive in place of sutures to treat rabbit's eyes following trabeculectomy (a routine procedure used to lower IOP in glaucoma patients) [114]. They recorded excellent biosafety and no sign of severe adverse effects from fibrin glues when compared with sutures. Based on these results, fibrin glues were considered a useful substitute for scleral and conjunctival wound closure procedures.

Fibrin-based glues have also been tested in the Pterygium surgery for conjunctival autografting [115-117]. In this context, fibrin glues were safe and patient friendly, with faster application than the use of suture fixation of grafts. Moreover, Kaufman et al. reported the satisfactory use of a fibrin adhesive for scleral amniotic patch adhesion and sutureless lamellar keratoplasty [68]. A fibrin-based adhesive was considered effective for sealing of conjunctival and skin grafts, LASIK flaps, and clear cornea incisions (Figure 5Av) [118]. Likewise, the combined use of a fibrin glue and amniotic membrane transplantation was demonstrated as a safe and effective method for repairing corneas with perforations of around $2 \mathrm{~mm}$ [119]. Complete reepithelization was reported after 15 days of the intervention, along with stable and healthy tissue recovery after 195-325 days.

The combined use of fibrin glues and stem cells has also been reported as a tissue engineering strategy for the repair of corneal tissues as an alternative to corneal transplantation [120-122]. For example, Rama et al. successfully accomplished re-epithelialization in 14 out of 18 ocular burn patients using this strategy [120]. The epithelium of the treated patients resumed a normal and 
transparent appearance after one month of the intervention, and the corneal tissue was healthy and stable after 12-27 months. Recently, the use of fibrin glues for surgical reconstruction of the ocular surface after tumor removal was also reported [123]. These studies suggest future opportunities to further extend the scope of fibrin-based adhesives in ocular applications.

Fibrin glues have found multiple niches of application as ocular sealants; they are perceived by many surgeons to be an effective alternative for the treatment of ocular lacerations [104] due to their practicality and clinical benefits including fast and practically complete re-adsorption. However, fibrin-based adhesives still face some outstanding challenges, mainly related to their batch-to-batch product variations, the possible presence of viral contamination, and immunological problems when derived from animal sources. This last issue has been addressed by the use of fibrin glue prepared from autologous blood [120]. However, the autologous approach itself presents disadvantages in terms of cost, time, and reproducibility/consistency of product properties.

\subsubsection{Serum Albumin-based Adhesive}

Albumin is another type of natural protein that has been widely investigated for the development of tissue sealants. Serum albumin (SA), the most abundant protein in mammalian blood, is a globular and water-soluble protein with a molecular weight in the range of 66.5-65.0 kDa. Human serum albumin (HSA) is a 585 aminoacids protein that contains 17 pairs of disulfide bridges and one free cysteine [124]. The molecular 3D structure of HSA has also been resolved (Figure 5Bi) [125].

Albumin-based materials have been investigated for cornea repair purposes. For example, BioGlue ${ }^{\circledR}$ (Cryoline Inc.) [10], a commercially available albumin-based adhesive that approved as a sealant for cardiac surgery by the FDA in 1999, has also been proposed to use for ophthalmic purposes. BioGlue ${ }^{\circledR}$ is a formulation that contains of $10 \%$ glutaraldehyde and $45 \%$ bovine serum 
albumin (BSA). Glutaraldehyde promotes covalent bonds between the lysine residues of albumin and the tissue, thus providing excellent mechanical properties (Figure 5Bii) [126]. However, several risks are associated with the use of this product. First, unreacted glutaraldehyde can introduce toxicity [127]. The SA (normally extracted from animal sources) may also present immunological risks. The use of recombinant versions of HSA (e.g. produced in Pichia pastoris) may help overcome safety concerns and reduce batch-to-batch variability of albumin-based sealants [128, 129].

In another study, Khadem et al. [130, 131] used laser-crosslinkable albumin-based formulations (laser soldering) to close cornea incisions. These materials were formulated with BSA and two different dyes (chlorin-e6 and Janus Green) that could trigger crosslinking between albumin in the glue and proteins in the ocular tissue upon photodynamic activation using $665 \mathrm{~nm}$ laser (Figure 5Biii). The adhesive was applied to rat corneal incisions in vivo (Figure 5Biv) and evaluated ex vivo at different times (1-14 days postmortem) to determine leakage pressure and inflammation by histological analysis. One day after application, the average leakage pressures were $430 \mathrm{mmHg}$ for the adhesive made from BSA/Janus Green glue, which was higher than that of the BSA/chlorine6 glue (357 mmHg). Likewise, the breaking strength was significantly higher for the BSA/Janus Green glue $(c a .3 .5 \mathrm{~N})$ than for its chlorin-e6 counterpart ( $c a .2 .5 \mathrm{~N}$ ) when the materials were used to glue rat skin and then submitted to tensile tests (Figure $5 \mathbf{B v}$ ).

Recently, the effective application of temperature control and radiometry during laser soldering to treat corneal cuts has been reported [132, 133]. For example, Tal et al. demonstrated the application of a temperature-controlled $\mathrm{CO}_{2}$ laser soldering system (power density of 16 $\mathrm{W} / \mathrm{cm}^{2} ; 65^{\circ} \mathrm{C}$ ) using a BSA-based solder (47\%) for corneal cut closure [132]. The authors used pigs as the animal model and demonstrated that the soldered corneas had milder stromal inflammation, less neovascularization, and a higher level of re-epithelization than sutured controls. 
The incorporation of nanoparticles in BSA has also been reported to produce laser solders with enhanced bioadhesive performance [134, 135]. However, the use of these materials has not yet reported for ophthalmic purposes. The mechanism underlying the laser soldering of proteinbased adhesives is not fully understood; mechanistic explanations include thermal remodeling, protein denaturation, and protein degradation, among others [136].

Altogether, the accumulated experimental evidence suggests that albumin-based adhesives could be serve as a suitable family of materials for ophthalmic use. However, some challenges to overcome remain the batch-to-batch product variations, and biosafety issues related to albumin obtained from animal sources.
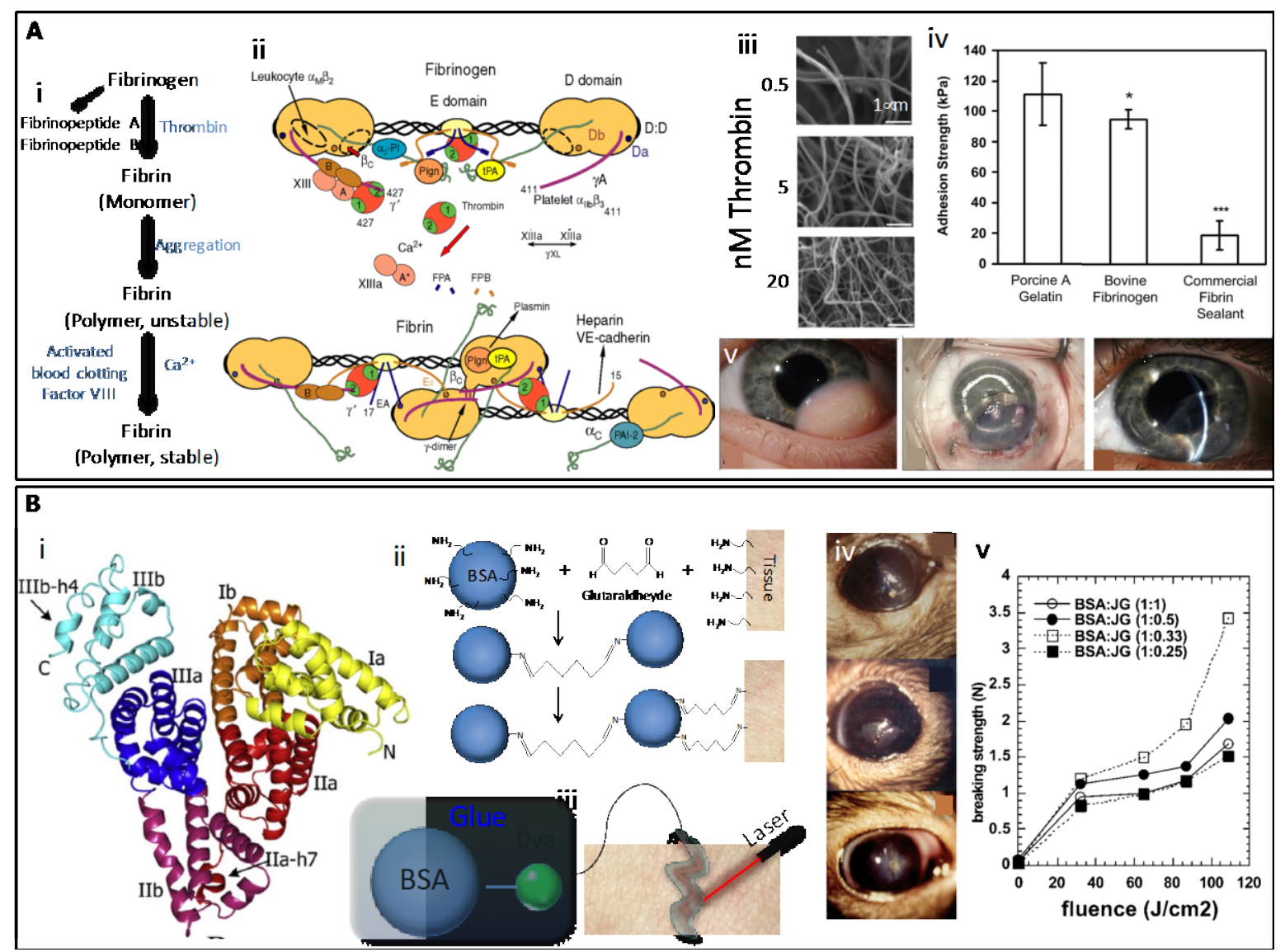

Figure 5. Structure, properties, and applications of fibrin- and albumin-based adhesives. (A) Mechanism of fibrin clot formation: (i) Schematic representation of the conversion from fibrinogen to fibrin and subsequent polymerization/crosslinking mechanisms [133], (ii) Fibrinogen structure and its thrombin-mediated conversion to 
fibrin. Binding sites for the main molecular actors that participate in fibrinogen functions are illustrated. Adapted from Mosesson et al. [109] with permission from Wiley, copyright 2005, (iii) Fibrin clots resulting from the addition of thrombin $(0.5-20 \mathrm{nM})$ solutions to fibrinogen $(2 \mathrm{mg} / \mathrm{mL})$ as observed by SEM. (Scale bar: $1 \mu \mathrm{m})$. Adapted from Wolberg et al. [110] with permission from Elsevier, copyright 2007, (iv) Comparison of adhesive strengths of photocrosslinked porcine gelatin, photocrosslinked bovine fibrinogen, and a commercial fibrin tissue sealant (Tisseel). Adapted from Elvin et al. [137] with permission from Elsevier, copyright 2010, (v) An application of fibrin glue in ophthalmology. Left eye of an infant patient with an inferotemporal growth before surgery, graft after attachment with fibrin adhesive, and 10 weeks later. Adequate graft integration with no edema and minimum haze was observed. Adapted from Zhou et al. [118] with permission from Healio, copyright 2016; (B) Albumin based adhesives: (i) The 3D structure of albumin; a globular protein abundantly present in animal serum, (ii) Mechanism of crosslinking and tissue adhesion of BioGlue ${ }^{\circledR}$, (iii) Schematic illustration of laser soldering using albumin-based solders. Adapted from Chao et al. [126] with permission from Wiley, copyright 2003, (iv) Rat eyes glued with albumin soldering after corneal epithelium removal surgery, and (v) ex-vivo breaking strength measure on mouse skin soldered with albumin-based solder. The ratio of protein to fluorescent dye has a significant effect on breaking strength as measured by tensiometer. Adapted from Khadem et al. [131] with permission from Wiley, copyright 2004.

\subsubsection{Collagen and Gelatin-based Adhesives}

Collagen is the most abundant protein in mammals, and the main structural protein in the extracellular microenvironment of the connective tissues in animals. Indeed, it is the most abundant protein in mammals. Not surprisingly, collagen-based materials have been investigated for the development of novel corneal adhesives. The highly complex structure of collagen has been, and continues to be, an important subject of study (Figure 6Ai-ii) [138]. Collagen is also naturally present in corneal stroma. It imparts very important functional properties to the cornea, including transparency and mechanical strength [139]. The architecture and properties of collagen fibrils have been extensively studied elsewhere [139-141].

The natural chemical properties of the endogenous corneal collagen have been exploited to correct keratoconus, a progressive eye disease that involves the bulging of the cornea into a cone shape. Since the early 90s, multiple reports have described different strategies to induce the crosslinking of the collagen originally presented in the cornea using riboflavin and UV-A irradiation. The 
effectiveness and safety of this general approach have been validated by several studies [142-145]. Avedro, Inc. was granted FDA approval for this technique to treat progressive keratoconus [146]. Later, the crosslinking procedure was accelerated from $1 \mathrm{~h}$ to $3 \mathrm{~min}$ by increasing the UV-A power. Recently, Richoz et al. observed that the process of corneal collagen crosslinking with riboflavin and UV light was oxygen dependent. Stronger crosslinking was achieved under high oxygen tensions [147].

Exogenous sources of collagens (animal-derived and recombinant collagen) have also been formulated in different ways to produce ophthalmic glues, e.g. films or coatings $[8,9,148]$, soldering [149], hydrogels [150], and vitrigels [151]. Collagen-coated surfaces exhibit appropriate as substrates for the adhesion and proliferation of corneal epithelial cells. For instance, Kim et al. documented the use of transparent poly(lactic-co-glycolic acid) (PLGA) films coated with collagen type I as a substratum for corneal tissue regeneration or inclusively as a material for artificial corneas for transplantation [148]. The authors demonstrated that primary corneal rabbit cells adhered, grew, and retained their expected cell morphology and phenotype when seeded in vitro on PLGA films coated with collagen, whereas they did not show these features when grown on pristine PLGA films without coating.

Collagen has been formulated as a soldering material (laser-crosslinkable) for corneal repair in a few reports [144, 149]. For example, Noguera et al. developed a collagen-based, laseractivated solder to repair corneal wounds [149]. Solder patches, fabricated by casting a concentrated solution of chemically modified collagen, followed by freeze drying, were used to repair incisions of 2 and $3 \mathrm{~mm}$ made ex vivo on rabbit corneas. The chemically modified collagen, used to fabricate the patches was prepared by reacting bovine or porcine collagen I with glutaric anhydride. The free amines within the collagen chain reacted with the anhydride to produce a 
material that underwent a thermal transition between 40 and $45{ }^{\circ} \mathrm{C}$ and had a sufficient number of carboxylic groups to crosslink with the cornea tissue upon exposure to low intensity laser light. The use of soldering patches rendered better results than conventional suturing. The IOP (125.16 $\pm 9.85 \mathrm{mmHg}$ ) was significantly higher in eyes treated with solder films than in sutured eyes (33.44 $\pm 9.38 \mathrm{mmHg})$.

Other collagen-based adhesives used for ocular repair include collagen with transglutaminase, which yields a crosslinked network by forming bonds between amine and acyl groups [152] (Figure 6Aiii), collagen-immobilized vinyl alcohol scaffolds to support corneal epithelium growth [153], collagen-based hydrogel scaffolds [150, 154], PEG-stabilized carbodiimide crosslinked collagen-chitosan hydrogels [155], alginate microsphere-collagen hydrogels [156], recombinant collagen versions [157], collagen-phosphorylcholine interpenetrating network hydrogels [158], collagen- and glycopolymer-based hydrogel [159], and collagen hydrogels crosslinked with carbodiimides [160].

In the last decade, collagen vitrigels (CV) have been also studied as scaffolds for different tissues, including eye tissues. Collagen vitrigels are thin transparent membranes made of highly compacted collagen I fibrils that resemble the natural architecture of collagen in the cornea [158]. Several reports have demonstrated that these materials are biocompatible and have good mechanical, optical, and permeability properties $[158,160,161]$. CVs are made by a three-step process: (1) gelation, (2) vitrification, and (3) rehydration. During the vitrification process, the collagen fibrils of the hydrogel compact together forming a dense network, rendering a rigid material, which is later rehydrated to obtain a soft and strong membrane. An optimized process to produce CVs with improved characteristics and shorter preparation/vitrification time was reported by Calderón-Colón et al. (Figure 6Bi) [151]. The authors tested conditions that produced CVs 
with a denaturation temperature of $54{ }^{\circ} \mathrm{C}$, which made them suitable for use at body temperature without risk of degradation. The vitrification conditions, including temperature, relative humidity (RH), and the vitrification time, had a clear influence on the collagen fiber self-assembly process and the vitrigel microstructure, which subsequently influenced the mechanical, thermal, and optical properties of the resulting CVs. Low vitrification temperature and short vitrification time resulted in a less compact microstructure. The collagen fibrils formed a tighter and more organized microstructure at higher temperature and longer vitrification time (Figure 6Bi). For instance, a $0.05 \mathrm{~mm} \mathrm{CV}$ vitrified at $10{ }^{\circ} \mathrm{C}$ with $20 \% \mathrm{RH}$ for half a week showed an open/loose mesh microstructure that exhibited an ultimate tensile strength of $669 \mathrm{kPa}$ and a $71 \%$ transmittance. By contrast, a CV vitrified at $40{ }^{\circ} \mathrm{C}$ with $40 \% \mathrm{RH}$ for 1 week showed a tighter and finer microstructure that exhibited an ultimate tensile strength of $8174 \mathrm{kPa}$ and an $84 \%$ transmittance. Guo et al. studied the effects of different vitrification conditions, including temperature, temperature changes, relative humidity, and vitrification time, on the final properties of CV membranes [162]. The process variables significantly influenced the nano-architecture of the membranes. Consistently, the authors observed that a high temperature $\left(39^{\circ} \mathrm{C}\right.$ ) and long vitrification time (8 weeks) resulted in CVs with highly dense structure (80 nm fibril-fibril space). In turn, the nano-architecture of CV membrane has a strong effect on the morphology and phenotype of keratocytes cultured on these CV membranes. When compared with growth on regular culture plates, keratocytes cultured on CVs exhibited a higher degree of branching with a longer average branch length [162]. In vitro studies with primary cell lines also suggested that CV membranes were potential candidates for reconstruction of the endothelial, epithelial, and stromal layers of the cornea [161].

Chae et al. evaluated the combined use of CV and chondroitin sulfate hydrogels to repair severe eye injuries in the battlefield. Treated injuries ranged from 3 to $8 \mathrm{~mm}$ in size from the cornea 
to the scleral region [9]. The authors used a "patch and glue system”, consisting of a pre-shaped (flat or curved) CV and a chondroitin sulfate adhesive hydrogel. Mechanical burst tests in porcine eyes, which consisted of injecting saline solution into the treated eyes at $20 \mathrm{~mL} / \mathrm{h}$ until a $35 \mathrm{mmHg}$ IOP was reached, revealed that the combined use of CV membranes and chondroitin sulfate glue was suitable for repairing wounds as large as $6 \mathrm{~mm}$ in superficial injuries and $8 \mathrm{~mm}$ in a deeper region (scleral). The glue itself (without the patch) was only able to repair superficial lacerations of $3 \mathrm{~mm}$ and lacerations at the scleral region of $4 \mathrm{~mm}$ before burst tests failed.

Chae et al. reported the use of CVs along with fibrin glue to repair corneas in limbal stem cell deficiency (LSCD) and stromal wound [163]. Both clinical conditions were induced in rabbits by keratectomy and by chemical burning, followed by corneal epithelium scraping, respectively. The application of CV glued with fibrin, CV with human limbal epithelial cells (hLEC), and fibrin glue only, were studied as different strategies to repair the tissue. The most successful results were observed with the CV and fibrin glue combination to repair stromal wounds, and CV with hLEC to treat LSCD (Figure 6Bii). In particular, the combined application of CV with fibrin glue allowed healthy regeneration of the corneal epithelium. In contrast, fibrin alone resulted in a hypertrophied corneal epithelium. Similarly, the strategy of using CV with hLEC allowed the growth of healthy and transparent epithelium, with low vascularization and inflammation, whereas vascularization and inflammation were significant in the control (untreated eyes) [163].

Gelatin is another promising candidate material for ophthalmic applications since it is derived from a partial hydrolysis of collagen, which is an endogenous component in the cornea. Gelatin is a water-soluble polypeptide mixture that can be crosslinked by different methods to produce adhesives and hydrogels with suitable properties for ocular tissue repair. Gelatin-microbial transglutaminase (gelatin-mTG) has been suggested as a suitable material for adhesive ophthalmic 
applications (Figure 6Aiii) [164-166]. Yamamoto et al. inferred vitrectomy with artificial posterior vitreous detachment (PVD) followed by retinal tear and detachment to rabbits [164]. Then, the authors used a Gelatin-mTG complex to treat the animals. After administration, the material adhered and sealed retinal tears for several days without noticeable inflammatory reaction (Figure 6Ci-iv). Chen et al. conducted additional in vitro adhesion studies on bovine retinal tissue using a similar gelatin-mTG biomimetic material and reported lap shear strength at wet conditions ranging from 15 to $45 \mathrm{kPa}$ [166]. These values were comparable to those reported for other softtissue glues, suggesting that mTG-crosslinked gelatin may be a suitable adhesive for ophthalmic applications. Sealants based on chemically modified gelatin, e.g. gelatin methacryloyl (GelMA) [167-169], are emerging players in eye tissue repair applications [170, 171]. For example, GelMA membrane crosslinked by dehydration $\left(160^{\circ} \mathrm{C}\right.$ for 6 to $48 \mathrm{~h}$ at 0.01 Torr) exhibited a comparable or superior performance versus commercially available collagen sheets (KOKEN Co. Ltd., Tokyo) as corneal endothelial cell carriers for corneal transplantation [170]. Gelatin sheets outperformed collagen sheets in many characteristics such as permeability, flexibility, transparency (Figure 6Cv), and elasticity. However, the toughness of gelatin formulations was lower than those measured for atelocollagen (enzymatically treated collagen) membranes (Figure 6Cvi). A healthy and ordered monolayer of corneal endothelial cells, attached to the gelatin hydrogel membranes, exhibited normal expression levels of sodium and calcium ATPases, ZO-1, and N-cadherin. 

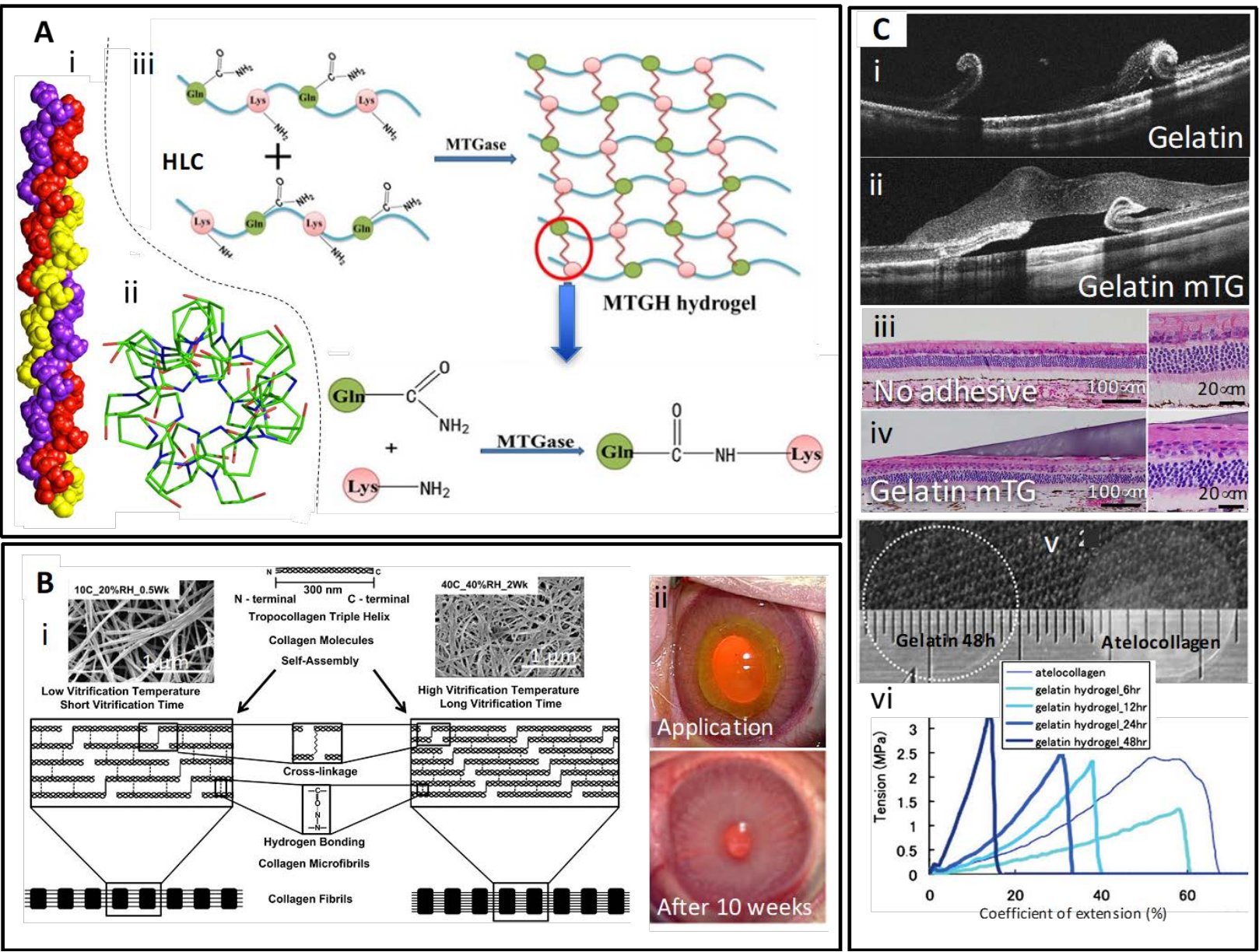

Figure 6. Structure, properties, and applications of collagen and gelatin-based adhesives. (A) Collagen-based

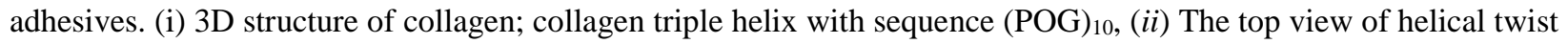
in the collagen structure. The top view of the T3-785 peptide (crystal structure), represents the first three POG triplets on each chain. Adapted from Bella [138] with permission from Portland Press, copyright 2016, (iii) Schematic representation of the mechanism of crosslinking of collagen (or gelatin) in the presence of transglutaminase. Transglutaminase enzyme forms amide bonds between the acyl groups in glutamine and the amino groups in lysine present in the protein (collagen or gelatin) chains. Adapted from Zhao et al. [152] with permission from Elsevier, copyright 2016; (B) Collagen and vitrigels. (i) Schematic explanation of the effect of vitrification temperature and time on the microstructure of collagen vitrigels. Insets are SEM images of the corresponding collagen vitrigels. Adapted from Calderón-Colón et al. [151] with permission from Elsevier, copyright 2012, (ii) Rabbit eye model with stromal wound treated with collagen vitrigel membrane and fibrin glue. Adapted from Chae et al. [163] with permission from Wiley, copyright 2015. (C) Gelatin-based adhesives. Optical coherence tomography (OCT) of a rabbit eye with retinal detachment treated (i) with gelatin or (ii) gelatin crosslinked with transglutaminase as observed 3 days of application. Images of histology transversal cuts of (iii) untreated and (iv) treated eyes three days after treatment. Gelatin-mTG adhesives tightly adhered to the retinal surface. Adapted from Yamamoto et al. [164] with permission from Springer, copyright 2013, (v) Transparency of gelatin and atelocollagen films after under wet 
conditions, (vi) Strain vs stress curves for different gelatin hydrogel films. Adapted from Watanabe et al. [170] with permission from Mary Ann Liebert, copyright 2011.

\subsection{Polysaccharide-based Adhesives}

Polysaccharides represent various biopolymers that are ubiquitously found in living organisms. Polysaccharides are linear or branched polymers that are constructed from different monosaccharides as their structural building blocks. The combination of sugar monomers, variations in functional groups, and selection of chemical linkages offer nearly unlimited deviations in chemical structures and biochemical properties of polysaccharides species. Common polysaccharide products extracted from animals or plants are regarded as biocompatible and biodegradable, therefore leading to widespread applications of polysaccharides in pharmaceutical, cosmetic, food, and biomedical industries. In general, polysaccharide-based tissue adhesives are hydrogels made from chemically-modified, water-soluble polysaccharides. Depending on the selection of polysaccharides and the desired way of delivery, crosslinking mechanisms of polysaccharide-based hydrogels may vary. In particular, researchers have focused on three specific kinds of natural polysaccharides to develop tissue adhesives for ophthalmic surgery applications, namely, chondroitin sulfate, dextran, and hyaluronic acid, which will be summarized in the following sections.

\subsubsection{Chondroitin-based Adhesives}

Chondroitin sulfate is a linear sulfated glycosaminoglycan composed of alternating $\mathrm{N}$ acetylgalactosamine and glucuronic acid units. Introduction of the sulfate groups can happen at different sites along the chain with varied degree of functionalization. In the living body, chondroitin sulfate is an important component of the cartilage tissues. The long-term biosafety of chondroitin 
sulfate has been well documented. As a result, it is widely used as a dietary supplement to prevent and treat osteoarthritis, although its medical effects are not clinically approved.

Several different chemical modification methods have been reported to prepare chondroitin sulfate-based hydrogels targeting at different biomedical applications. For example, Li et al. reported the mild reaction between chondroitin sulfate and glycidyl methacrylate to synthesize a photocrosslinkable, methacrylated chondroitin sulfate derivative [172]. By changing parameters such as degree of functionalization, prepolymer concentration, and crosslinking conditions, chondroitin sulfate hydrogels with tunable physical properties could be fabricated by facile photopolymerization techniques. Moreover, cytocompatibility of the hydrogel was established by encapsulation experiments of chondrocytes, which remained viable and metabolically active, thus suggesting potential applications in cartilage tissue engineering [114]. 

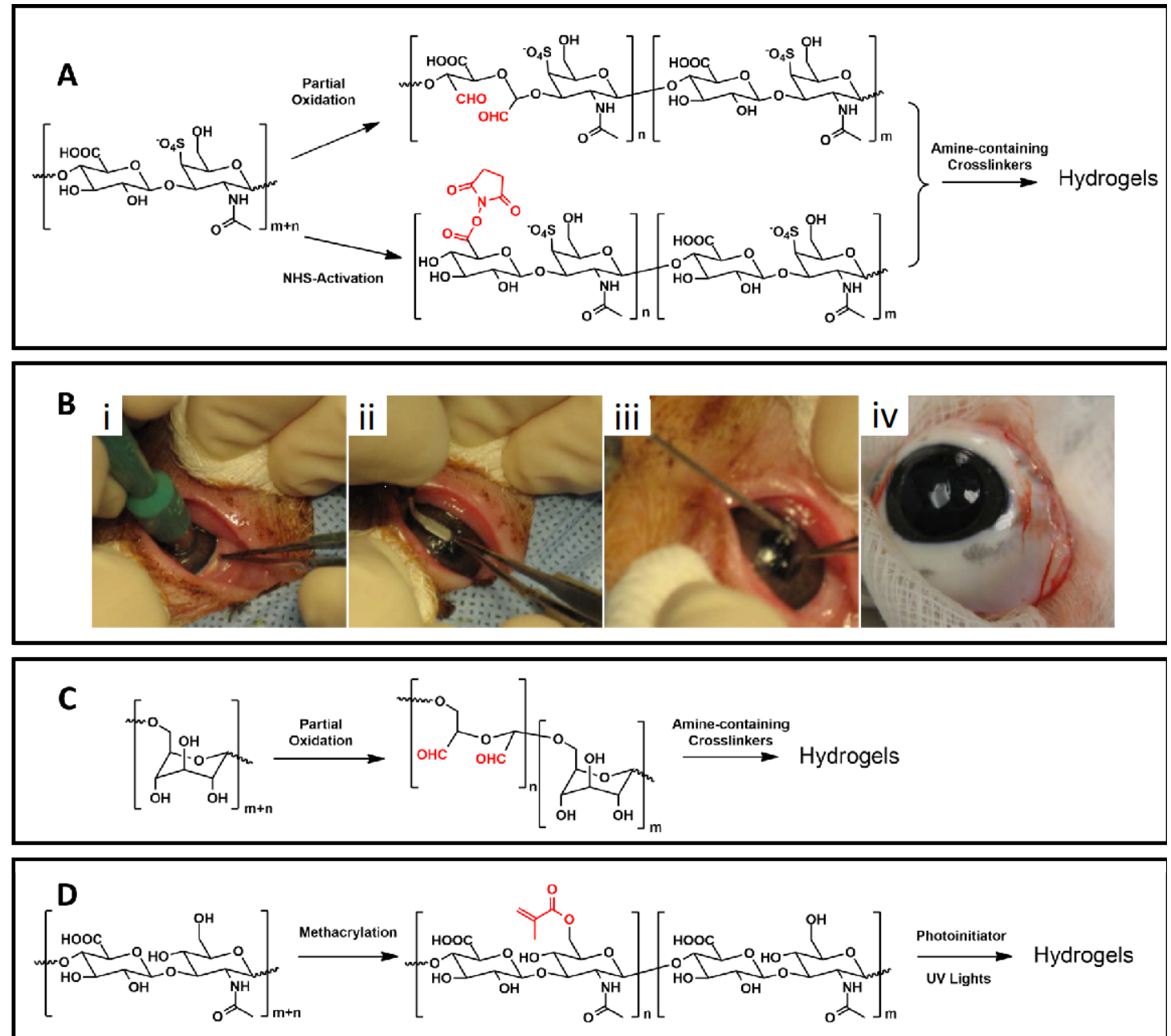

Figure 7. Examples of polysaccharide-based adhesives. (A) Commonly used chemical crosslinking mechanisms for chondroitin sulfate-based hydrogels. (B) Application of NHS-modified chondroitin sulfate/amine PEG sealants in a swine eye model, (i) A $6.0 \mathrm{~mm}$ defect was made in the cornea with a trephine, (ii) an incision was made to get a flap, (iii) the sealant was applied to glue the flap to the stroma, (iv) the flap was tightly adhered 2 weeks after surgery. Adapted from Strehin et al. [173] with permission from Elsevier, copyright 2009. (C) Schematic illustration of the partial oxidation of dextran and the formatting mechanism of hydrogels crosslinked by amine-containing crosslinkers. (D) Schematic illustration of the methacrylation and photocrosslinking reactions of hyaluronic acid-based sealant.

Reyes et al. reported another crosslinking chemistry based on the controlled oxidation of chondroitin sulfate by sodium periodate to introduce reactive aldehyde groups, which form crosslinked networks with amine groups by formation of Schiff base linkages (Figure 7A) [174]. The usage of chondroitin sulfate hydrogel, as an adhesive layer between the implanted biomaterials 
and native cartilage tissues, showed strong adhesion and stable integration (over five weeks in vivo) to repair wounded cartilage tissues [175]. NHS-activated chondroitin sulfate derivative has also been reported by Strehin et al., which could react with amine-bearing PEG crosslinkers to form stable hydrogels with amide linages (Figure 7A). In addition, the NHS-ester groups could also react with amine groups on tissue surfaces to enhance adhesion $[173,176]$.

An ophthalmic adhesive based on chondroitin sulfate aldehyde and polyvinyl alcohol covinylamine (PVA-A) was formulated to seal corneal incisions [174]. As discussed above, the aldehyde groups can react with amine groups in PVA-A to generate hydrogels in situ. The efficacy of this chondroitin sulfate-based adhesive was compared with standard suture techniques to seal uniplanar, clear corneal incisions (3 mm) in an ex vivo rabbit model [174]. The maximum IOPs before wound leakage of the sealed or sutured eyes were evaluated and compared to reveal the effectiveness of the adhesive. The mean IOP observed in glued eyes was $101.4 \pm 3.2 \mathrm{mmHg}(\mathrm{n}=8)$, which was significantly higher than those values from either single-suture or three-suture subgroups (26.4 \pm 6.0 and $44.3 \pm 8.2 \mathrm{mmHg}$, respectively). This indicates that the chondroitin sulfate-based adhesive is a promising material to replace sutures as a corneal incision sealant [174]. Another chondroitin sulfate based adhesive was also tested in the microkeratome-assisted posterior lamellar keratoplasty setup to evaluate the astigmatic change and the stability of the graft stability when using the tissue adhesive in the modified procedure [177]. The standard suture technique was used as control. A partial flap keratectomy (300- $\mu \mathrm{m}$-thick) was conducted ex vivo in corneoscleral rims of a human donor with a 6.25-mm trephination, which was subsequently fixed with either five interrupted sutures or the chondroitin sulfate-based glue [177]. It was revealed that both techniques showed comparable mean astigmatic changes ( $3.08 \pm 0.84 \mathrm{D}$ for sutures $v s .1 .13 \pm 0.55 \mathrm{D}$ for the adhesive) and similar mean resistant pressures (95.68 $\pm 27.38 \mathrm{mmHg}$ for suture vs. $82.45 \pm 18.40 \mathrm{mmHg}$ for 
the adhesive). The results indicated that these suture-less alternatives are promising for clinical treatment of corneal endothelial disorders [177].

The ability to seal small corneal incisions of the NHS-activated chondroitin sulfate/aminePEG adhesive was also accessed in a swine model (Figure 7B). Tested on a 6.0-mm defect made in the swine cornea, this chondroitin sulfate-based adhesive was able to restore maximum IOP greater than 200 mmHg. The adhesive was also non-toxic to major types of cells found in the cornea. Moreover, histological results demonstrated minimal inflammatory response and no scar formation after two weeks of application $[173,176]$. Taken together, these results confirmed that chondroitin sulfate-based adhesives are promising candidates for ophthalmic applications. The excellent biosafety of chondroitin sulfate, superior surgical performance, and the versatile chemistry for tailored properties of the adhesives are the desirable advantages of chondroitin sulfate-based tissue adhesives. However, since the most common source for producing chondroitin sulfate is from animal tissues, concerns associated with long-term biosafety and batch-to-batch variation should be noted for future clinical applications.

\subsubsection{Dextran-based Adhesives}

Dextran is a complex polysaccharide with certain branches along the linear chain. The main linear section is composed of glucose linked by alpha-1,6 bonds. As dextran lacks multiple reactive substitutional groups (e.g. amine groups and carboxylic acid groups), the availability of functional groups for chemical modifications are limited. The most common chemical modification strategy of dextran is based on a controlled oxidation reaction by periodate, similar to that of chondroitin sulfate (Figure 7C). Subsequently, the oxidized aldehyde-containing dextran derivatives can react with amine-bearing crosslinkers to form hydrogels via the imine linkage formation. Other strategies 
include photocrosslinkable dextran derivatives with methacrylate groups [178] and enzymeresponsive dextran materials with tyramine motifs [179].

Araki et al. developed a dextran-based tissue sealant by reacting aldehyde-bearing dextran with $\varepsilon$-poly(L-lysine) [180, 181]. The adhesive properties of this sealant were first evaluated in an in vivo lung model using fibrin glues as the controls. Biodegradability and biocompatibility of this adhesive were also evaluated in a dog model with showed the potential of the tissue adhesive [180, 181]. Later, this aldehyde dextran/e-poly(L-lysine) was applied as the adhesive in suture-less amniotic membrane transplantation to fix the membrane to the ocular surface in rabbit model [182]. In vivo experiment results showed that the dextran-based adhesive could tightly fix the membrane to ocular surface, did not interfere with the cell growth, and promoted tissue regeneration. Moreover, the adhesive material could be degraded in 4 weeks in vivo, therefore providing a valuable alternative to replace sutures and reduce the risks of suture-related infection, scarring, and damage to surrounding tissues [182].

Dextran-based ophthalmic adhesives were also tested in a suture-less automated lamellar therapeutic keratoplasty setup to evaluate their ability to fix grafts [183]. A three-month study in an in vivo rabbit model proved that grafts sealed to the cornea remained attached and clear for 90 days. Epithelialization on the glued graft was observed within 7 days with no indication of apparent signs for inflammation or scarring. This study demonstrated that dextran-based adhesives can be an alternative technique in treating corneal diseases [183]. Bhatia et al. documented the use of a similar dextran-based tissue adhesive containing a crosslinker consisting in an 8-arm amine-PEG derivative [184]. The cytotoxicity of this adhesive was first tested in vitro using J774 macrophage cells and 3T3 fibroblast cells. Results confirmed that the dextran-PEG adhesive was not toxic to the cells and did not induce a significant inflammatory cell activation (J774 macrophages did not release 
inflammatory markers) [184]. The authors further evaluated the ex vivo sealing ability of the adhesive on $5 \mathrm{~mm}$ corneal incisions in a rabbit model. By applying $40 \mu \mathrm{L}$ of the adhesive prepolymer solution, the sealed incision could resist pressures up to $557 \pm 23 \mathrm{mmHg}$, providing promising implications that these could be used to replace suture procedures in closing corneal wounds [185]. The authors later reported an improved delivery device to reduce the volume of adhesive needed for sealing a $3.2 \mathrm{~mm}$ corneal incision to less than $2 \mu \mathrm{L}$. The sealed corneal could resist leakage pressure of at least $120 \mathrm{mmHg}$ for at least 5 days post application [186].

The dextran-based ophthalmic adhesives have been the subject of multiple in vitro and in vivo studies to demonstrate their excellent performance in closing corneal wounds and fixing grafts to the ocular surfaces. Their biocompatibility has been examined by histological experiments to prove that the adhesives did not induce inflammation nor interfere with tissue regeneration. However, dextran-based adhesives offer less potential to tailor their properties in comparison to other molecules (i.e., PEG-based, dendrimer-based and chondroitin-based adhesives) because of their scarcity of functional groups available for chemical modifications.

\subsubsection{Hyaluronic Acid-based Adhesive}

Hyaluronic acid (HA) is a polysaccharide composed of 2-acetamide-2-deoxy-D-glucose and D-glucuronic acid units. HA is a natural component of the gel filling in eyes and has also been correlated with cellular migration behavior in the wound healing process. As a result, HA is a very appealing material for the development of tissue adhesives or scaffolds for tissue engineering applications. For ocular applications, HA-based adhesives can be synthesized based on a photocrosslinkable derivative. 
Miki et al. reported the chemical modification of HA with methacrylic anhydride to introduce methacrylate groups onto the polysaccharide backbone (Figure 7D) [8]. The resulting methacrylated hyaluronic acid (HAMA) was combined with the ethyl eosin and triethanolamine photoinitiator system to formulate the photopolymerizable hyaluronic acid-based adhesive. A low-density argon laser $(514 \mathrm{~nm}, 200 \mathrm{~mW})$ was used to convert the viscous liquid prepolymer solution into a crosslinked transparent hydrogel network. In an in vivo rabbit model, the prepolymer solution was delivered to the $3 \mathrm{~mm}$ corneal laceration and polymerized in situ to seal the incision. No leakage was observed in 37 out of 38 rabbit eyes and the seal lasted for at least 7 days. The HA-based adhesive did not show in vivo cytotoxicity nor inflammatory responses. Moreover, the deposition of new ECM and the proliferation of stromal cells at wound sites were observed at day 7 post-surgery, suggesting rapid tissue regeneration of the sealed cornea. The measured IOP values for this HA-based ocular adhesive were 5-10 mmHg by day 1 and increased to $10-15 \mathrm{mmHg}$ by day 7 . This study suggested that HA-based adhesives could also be developed for various ocular applications [8].

Despite the excellent biosafety and reported good results of HA-based adhesives, the use of photopolymerization technique in ophthalmic applications might raise concerns about light-induced tissue damage or about the relative complex experimental setups to introduce the light source to the operating room. Future developments to introduce other crosslinking mechanisms to hyaluronic acid-based hydrogels might expand the applications of these polysaccharides in ophthalmic adhesives.

So far, we have presented and discussed the characteristics, advantages and disadvantages of a spectrum of eye adhesives. Among several characterization methods, the leakage pressure measured on ex vivo eyes, have been used by several authors as a useful parameter to compare the performance of ocular sealants (Table 1). Other important parameters frequently reported include: 
cytotoxicity, inflammatory response, tissue regeneration, biodegradability, transparency, microstructure and other mechanical properties (in addition to leakage pressure). Patient comfort is one of the main aims of developing and using ocular adhesives, however, this is a "parameter" that is elusive to quantitation and has been less frequently reported in literature, except for cyanoacrylates or products already commercially available. Novel promising alternatives are being developed today, and in the years to come, they will hit the market place yielding benefits to physicians and patients. Some of these new materials are more than simply adhesives; they are engineered to provide benefits that go beyond adhering tissues and provide with additional features or "upgrades" (i.e., drug delivery or sensing capabilities) to the conventional concept of ocular adhesive.

Table 1. Summary of reported ocular adhesives and their efficiency in sealing an ocular leak.

\begin{tabular}{|c|c|c|c|c|}
\hline Category & $\begin{array}{c}\text { Leakage pressure } \\
\text { (mmHg) }\end{array}$ & Wound type & $\begin{array}{c}\text { Ex vivo } \\
\text { model }\end{array}$ & Refs \\
\hline Octyl 2-cyanoacrylate & $55.13 \pm 10.46$ & \multirow{2}{*}{$\begin{array}{l}\text { 5.2-mm full-thickness central } \\
\text { corneal lacerations on porcine }\end{array}$} & \multirow{2}{*}{ porcine } & \multirow{2}{*}{ [187] } \\
\hline Nylon $10-0$ suture & $52.37 \pm 7.16$ & & & \\
\hline$n$-Butyl cyanoacrylate & $51.2 \pm 15.0$ & \multirow{4}{*}{$\begin{array}{l}\text { 2.5-mm uniplanar corneal } \\
\text { incision }\end{array}$} & \multirow{4}{*}{ rabbit } & \multirow{4}{*}{ [53] } \\
\hline $\begin{array}{l}\text { Methoxypropyl } \\
\text { cyanoacrylate }\end{array}$ & $65.2 \pm 14.5$ & & & \\
\hline Fibrin glue & $17.5 \pm 5.2$ & & & \\
\hline Nylon $10-0$ suture & $15.6 \pm 5.2$ & & & \\
\hline $\begin{array}{l}\text { PEG(3400)-DA with } \\
\text { ([G2]- (Lys)3 -Cys4 ) }\end{array}$ & $184 \pm 79$ & \multirow{2}{*}{$\begin{array}{l}\text { 3-mm clear corneal linear } \\
\text { incision }\end{array}$} & \multirow[t]{2}{*}{ chicken } & \multirow[t]{2}{*}{ [91] } \\
\hline Suture group & $54 \pm 16$ & & & \\
\hline $\begin{array}{l}\text { PEG(3400)-DA with } \\
\text { ([G2]- (Lys)3 -Cys4 ) }\end{array}$ & $142 \pm 22$ & \multirow{2}{*}{$\begin{array}{l}\text { 2.75-mm clear corneal } \\
\text { cataract incisions }\end{array}$} & \multirow{2}{*}{ Human } & \multirow{2}{*}{ [188] } \\
\hline Non-sealed wound & $77 \pm 14$ & & & \\
\hline [G1]-PGLSA-MA)2 -PEG & $171 \pm 44$ & \multirow{2}{*}{ 4.1-mm linear laceration } & \multirow{2}{*}{ chicken } & \multirow{2}{*}{ [78] } \\
\hline Suture group & $90 \pm 18$ & & & \\
\hline [G1]-PGLSA-MA)2 -PEG & $109.6 \pm 82.7$ & \multirow{2}{*}{ 4.1-mm linear laceration } & \multirow{2}{*}{ Human } & \multirow{2}{*}{ [98] } \\
\hline Suture group & $78.7 \pm 27.8$ & & & \\
\hline CS-PEG + CV & 35 & 22-mm linear laceration & Porcine & [9] \\
\hline
\end{tabular}




\begin{tabular}{|c|c|c|c|c|}
\hline BSA-ce6 & $357 \pm 25$ & \multirow{3}{*}{$\begin{array}{l}\text { 6-mm full-thickness central } \\
\text { corneal incision }\end{array}$} & \multirow{3}{*}{ Rat } & \multirow{3}{*}{ [131] } \\
\hline BSA-JG & $430 \pm 36$ & & & \\
\hline Non-sealed group & $193 \pm 6$ & & & \\
\hline $\begin{array}{l}\text { Laser-soldering collagen } \\
\text { patch }\end{array}$ & $3.62 \pm 3.09$ & \multirow{3}{*}{$\begin{array}{l}\text { 3.0-mm uniplanar peripheral } \\
\text { oblique corneal wound }\end{array}$} & \multirow{6}{*}{ Rabbit } & \multirow{6}{*}{ [149] } \\
\hline Suture group & $33.44 \pm 9.38$ & & & \\
\hline Non-sealed group & $125.2 \pm 9.85$ & & & \\
\hline $\begin{array}{c}\text { Laser-soldering collagen } \\
\text { patch }\end{array}$ & $101.4 \pm 29.92$ & \multirow{3}{*}{ 2.85-mm straight keratome } & & \\
\hline Suture group & $82.7 \pm 6.55$ & & & \\
\hline Non-sealed group & $1.7 \pm 0.13$ & & & \\
\hline
\end{tabular}

\section{Adhesives for Ocular Drug Delivery}

The eye has various protective mechanisms, such as tear production, tear flow, and blinking, that promote rapid drainage of the drug via the nasolacrimal duct. Therefore, conventional strategies for ocular drug delivery, including the application of solutions and suspensions, can be partially ineffective since liquids can be readily washed from cornea through natural processes. Ointments have been used to deliver ocular drugs and overcome these challenges, but they blur vision by changing the tear's refractive index [189]. To address these limitations, drug delivery components have been incorporated into bioadhesives and soft contact lenses to extend the residence time of active ingredients in the eye [190]. Here, we review bioadhesives designed for various ocular applications, ranging from drug delivery to "wound-filling” where significant stromal loss in the cornea puts the eye at risk of perforation and thus needs to be closed for securing the anatomical integrity of the eye as well as promoting tissue healing and regeneration. It is important to note that some bioadhesives have inherent antibacterial properties, while others are granted therapeutic or protective properties by impregnating drugs in the bioadhesive or incorporating drug-loaded constructs, such as nanoparticles and microspheres [189, 190]. 
Giano et al. used a novel syringe-injectable, an inherently antibacterial bioadhesive hydrogel for wound-filling applications [190]. The bioadhesive had antibacterial properties against both Gram-negative and Gram-positive bacteria, without harming human erythrocytes. This bioadhesive was developed by mixing branched polyethylenimine and polydextran aldehyde and achieved a maximum adhesive stress of $2.8 \mathrm{kPa}$. Using a murine infection model, it was shown that this adhesive could kill Streptococcus pyogenes present on the bioadhesive's surface, without inducing much inflammation. In addition, the adhesive was investigated through a cecal ligation and puncture model and was found to inhibit sepsis [190].

One of the first examples of a drug-impregnated bioadhesive was provided by Hui and Robinson [191]. They synthetized a drug-loaded polymeric adhesive, in which the polymer consisted on acrylic acid crosslinked with 2,5-dimethyl-1,5-hexadiene and progesterone was used as a drug model. The progesterone was incorporated to the system during polymer synthesis. The material was swollen in water to form a tridimensional matrix poly(acrylic acid hydrogel) with an entrapped drug (progesterone) which was dissolved by the water within the hydrogel and would be released by diffusion. The swollen poly(acrylic acid) adhesive exhibited good bioadhesion to rabbit conjunctival membranes (231 \pm 44 dynes $\left./ \mathrm{cm}^{2}\right)$. In vitro release studies showed that the system did not offer enough barrier opposition to control the release rate of the entrapped progesterone. However, bioavailability studies using albino rabbits demonstrated a statistically significant increase of drug concentration in the aqueous humor in the animals that received the drug through the bioadhesive as compared to the ones that received it in suspension (drops) [191].

Chae et al. investigated the combination of a chondroitin sulfate-polyethylene glycol (CSPEG) adhesive and a corneal-shaped, collagen-based CV membrane as a means of treating penetrative ocular injuries on the battlefield [9]. The corneal-shaped CV successfully matched the 
corneal contour without wrinkling. A vancomycin-loaded CS-PEG component, used to control release antibiotics, shown to inhibit Staphylococcus infection for 9 days. The CS-PEG was found to treat wounds (5-mm to 6-mm length) in the cornea and corneoscleral regions. However, the combination of CS-PEG and CV was required to treat larger wounds [9]. In another study, it was shown that silver nanoparticles can reduce the growth of bacteria by at least one order of magnitude and enhance mechanical strength of the ocular adhesives [192]. The greatest mechanical strength was observed with adhesives doped $10 \mathrm{~nm}$ silver nanoparticles at a concentration of $10 \mu \mathrm{g} / \mathrm{mL}$; the force required to disrupt these adhesive bonds was approximately $22 \mathrm{kPa}$.
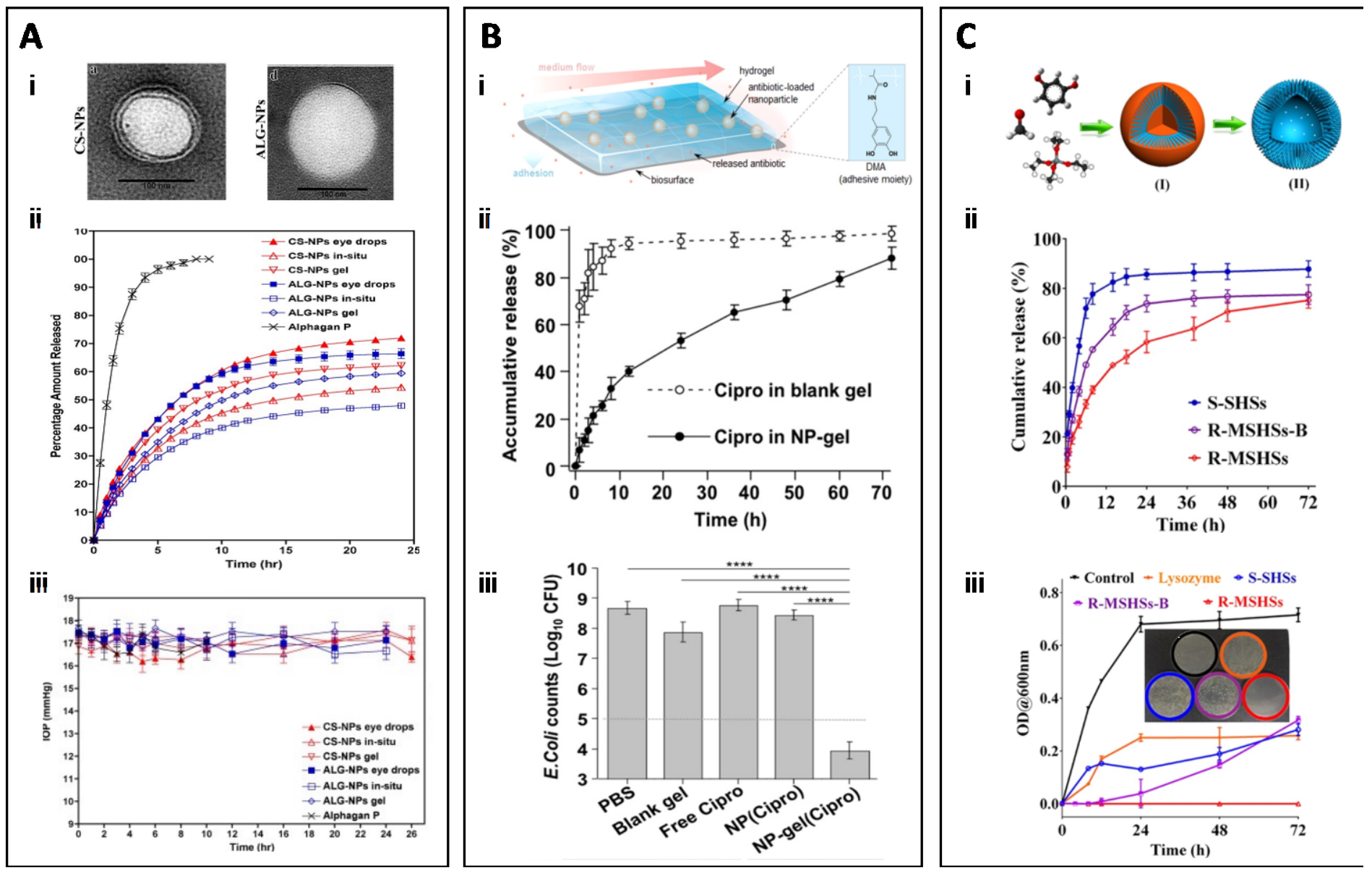

ii

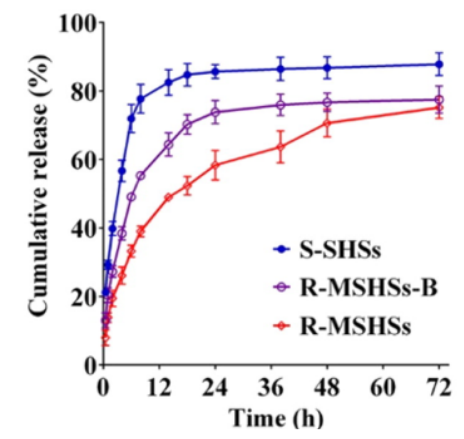

iii

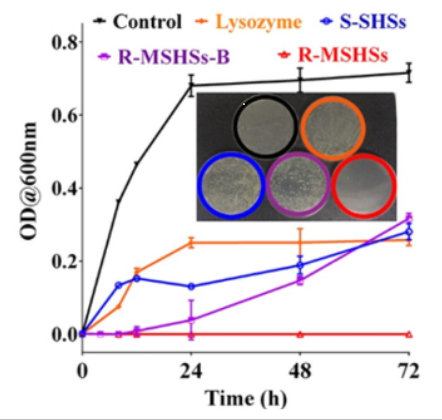

Figure 8. Examples of bioadhesives used for drug delivery. (A) A bioadhesive to lower the IOP. (i) TEM images of the brimonidine-loaded chitosan (CS) and alginate (ALG) nanoparticles, (ii) Release profile of brimonidine from the nanoparticles eye drops, gel and in situ, (iii) IOP effect of the formulation. Adapted from Ibrahim et al. [193] with 
permission from ARVO, copyright 2015; (B) (i) Schematic of the NP-gel system, consisting of ciprofloxacin-loaded nanoparticles in a 3D adhesive hydrogel, (ii) Release profile of ciprofloxacin in vitro, (iii) Effect of the adhesive on E. coli bacterial film in vivo. Adapted from Zhang et al. [194] with permission from American Chemical Society, copyright 2016; (C) (i) Schematic of the hollow nanocarriers loaded with lysozyme, with rough surface to enhance adhesion to bacteria, (ii) Release profile of lysozyme in vitro for smooth silica hollow spheres (S-SHSs), rough mesoporous silica hollow spheres (R-MSHSs), and rough mesoporous silica hollow spheres with blocked shell (RMSHSs-B) (iii) Antibacterial efficacy of the adhesive in vitro toward E. coli. Adapted from Song et al. [195] with permission from American Chemical Society, copyright 2016.

Drug delivery systems can be also designed by first incorporating drugs into nanoparticles (NPs) and then combining them with hydrogel prepolymer solutions. Chitosan and sodium alginate are natural and biodegradable polymers that have been commonly used in the preparation of NPs for ophthalmic formulations. Chitosan has unique properties such as bioadhesiveness, biocompatibility, biodegradability, and inherent antibacterial activity, which together make it an attractive mucoadhesive polymer [193]. In a study by Motwani et al. [196], chitosan and sodium alginate nanoparticles were explored for ophthalmic delivery of the antibiotic gatifloxacin for a prolonged time period [196]. Gatifloxacin, an antibacterial agent used to treat ocular infections, was released in vitro in a sustained manner for $24 \mathrm{~h}$, as compared with conventional eye drops which fail to achieve sustained drug release and often require a high dosing frequency. In another study, Ibrahim et al. used an adhesive that contained NPs of either chitosan or alginate, loaded with brimonidine (Celecoxib ${ }^{\mathrm{TM}}$ ), for the management of glaucoma (Figure 8A) [193]. The alginate NP were incorporated into a hydrogel formulation that consisted of in situ gelling system triggered by temperature and exhibited sustained release in vitro (Figure 8Ai-ii). Additionally, an in vivo study in mice revealed that the IOP-lowering effect of the formulation lasted for more than $25 \mathrm{~h}$ after application and thus achieved a sustained effect that was comparable to that of the commercially available Alphagan P eye drops (Figure 8Aiii) [193]. 
Zhang et al. loaded ciprofloxacin, a wide spectrum antibiotic, into PLGA NPs that were subsequently embedded into a 3D adhesive hydrogel [194]. The NP-gel was created by mixing the NPs with dopamine methacrylamide (DMA; adhesive agent and monomer), acrylamide (a monomer), poly(ethylene glycol) methacrylate (PEG-MA) (crosslinker), and lithium phenyl-2,4,6trimethylbenzoylphosphinate (photoinitiator) (Figure 8Bi). This hybrid system allowed for prolonged antibiotic release (Figure 8Bii) while achieving reliable adhesion under high shear stress (3.4 Pa). The adhesion properties of the NP-gel, under flow conditions, on different biological surfaces (mouse skin tissue, mammalian cell monolayers, and bacterial films) were evaluated and found to be superior in response than the gel without DMA. For example, in a peeling test where two layers of mouse skin were glued with $100 \mu \mathrm{L}$ of NP-gel adhesive, a force of around $0.3 \mathrm{~N}$ was required to separate the skin layers, as opposed to the $0.03 \mathrm{~N}$ required for the control, a non-adhesive NP-gel. The viscoelasticity and adhesion property could be tuned with regard to the shear stresses present in different conditions. Additionally, the hybrid composition was found to inhibit the development of a biofilm of E. coli under flow conditions in vitro (Figure 8Biii) [194].

Song et al. explored the potential of drug-loaded nanocarriers with a rough surface, inspired in the morphology of pollen grains, to enhance adhesion to bacteria and achieve long-term bacterial inhibition (Figure 8Ci) [195]. The rough mesophorous silica hollow spheres were loaded with lysozyme (an antibacterial lytic enzyme) and exhibited sustained release of the drug and inhibited E. coli growth for up to 3 days in vitro (Figure 8Cii). The nanospheres were also loaded with lysozyme, an antimicrobial enzyme, and showed a significantly higher antimicrobial activity compared with free lysozyme, which was not able to achieve the minimum inhibitory concentration of $700 \mu \mathrm{g} / \mathrm{mL}$ even at very high concentrations of $2 \mathrm{mg} / \mathrm{mL}$ (Figure 8Ciii) [195]. 
To study the antibacterial efficacy ex vivo, a rat small intestine infection model was used. The rough silica spheres exhibited more than two orders of magnitude lower E. coli count compared to phosphate buffer solution and free lysozyme administration, and 30 times lower count compared to smooth hollow silica spheres loaded with lysozyme (Figure 8Ciii) [195].

Bioadhesives have been also used for drug delivery through the incorporation of drug-loaded microspheres. Sensoy et al. created bioadhesive sulfacetamide sodium (SA) microspheres for effective delivery of ocular keratitis by increasing the bioavailability of the drugs on the ocular surface [197]. Spray drying was used to fabricate microspheres using different ratios of a combination of polymers with mucoadhesive properties, such as polycarbophil, hydroxypropylmethyl cellulose, and pectin, and drugs. The most suitable polymer-to-drug ratio for ocular applications was of 2:1. This polymer-drug ratio (suspended in light mineral oil) was used in vivo to treat bacterial keratitis on New Zealand male rabbit eyes infected by S. aureus or Pseudomonas aeruginosa. In both infection models, a significant decrease in bacteria count was observed in eyes treated with SA-microspheres versus those treated with free SA, illustrating the effectiveness of SA-loaded microspheres in the treatment of ocular keratitis [197].

A bioadhesive ophthalmic drug insert (BODI) was developed by Baeyens et al. [198]. The placement of this soluble insert in the lower cul-de-sac of the eye allows for prolonged release of gentamicin, which addressed the inconvenience associated with several daily applications of eye drops over a prolonged period of time that is normally required to treat ocular diseases. The clinical efficacy of BODI was compared with that of the classical eye drop treatment Tiacil on dogs. BODI was shown to be as effective as Tiacil in treating the symptoms of the external ophthalmic diseases, conjunctivitis and keratoconjunctivitis sicca, with the advantage of providing the treatment in a single application [198]. 
By creating novel and effective bioadhesives for drug-delivery, it is possible to overcome some of the setbacks associated with conventional ocular drug delivery systems such as solutions and suspensions. This section discussed ocular bioadhesives that are capable to achieve their desired physiological outcomes through their inherent properties or through the integration of drugs in their formulations. The use of micro- and nanoparticles in these formulations provides great flexibility to the design of drug-loaded ocular adhesives, by providing additional means to control (fine-tune) drug release and improve residence time, efficacy, or adhesive strength. In the years to come, adhesives used for applications other than ocular drug delivery, will be tailored towards ocular applications through simple design modifications.

\section{Bandage Contact Lenses and Prefabricated Patches}

Over the last decade, the number of contact lens (CL) users has tremendously increased, and currently over 70 million people worldwide wear CLs [199]. Bandage CLs, specifically, have been used over the last four decades in clinic after refractive surgery to aid patient recovery. They provide a physical barrier that allows the tissue to heal, and apply pressure to relieve pain [200]. Generally, the patient needs to continuously use soft bandage CLs after ophthalmic surgery, in order to sustain sufficient ocular surface hydration, protect the eye from necrosis, and improve comfort after surgery [201]. Silicon hydrogels are the most commonly used materials to fabricate CLs because of their softness (Modulus: 0.5-1.4 MPa), flexibility, water content (24-47\%), transparency, and high gas permeability (oxygen permeability, DK: 60-14) which are key characteristics for bandage CLs. Non-silicon CLs are also used after surgery. However, their use for extended period leads to tissue swelling due to their low gas permeability. Balafilcon A (Pure Vision, Bausch \& Lomb), Lotrafilcon A (Night \& Day, Ciba Vision), and Lotrafilcon B ( $\mathrm{O}_{2} \mathrm{Optix}$, 
CIBA Vision) are silicone hydrogels approved by the FDA for use as bandage CLs for extended period of time (6, 30, and 30 days after refractive surgery, respectively) [200, 202, 203].

CL technology has advanced at a rapid pace in various innovative directions, including as drug delivery systems [204, 205], delivery of antimicrobial agent [206], biosensors [199, 200], and electronic displays [199, 207]. These technologies will certainly substantially benefit the ocular wound care arena in the short term. Several examples of new technological functionalities incorporated into CLs are discussed in this section (Figure 9).

CLs have been widely studied as effective drug delivery systems. This is mainly due to limitations of traditional ophthalmic drug delivery methods, (e.g. topical delivery using eye drops), such as: low efficiency patient noncompliance, and poor ocular drug bioavailability (less than 5\%) [208]. Several groups have attempted to develop CLs for drug delivery applications using different strategies such as drug soaking [204, 209, 210], using vitamin E as a diffusion barrier [211-214], NP laden CLs (Lipid-based and polymers) [215-219], molecular imprinting [80, 220-222], and layer-by-layer platforms [223, 224]. For instance, Carreira et al. developed a novel bandage CL made from PVA and chitosan and incorporated with vancomycin using a "soak and release" technique to prevent inflammation after corneal substitution [225].

The incorporation of NPs in formulations such as microemulsions, liposomes, cyclodextrins and polymer-based NPs into the CL structure is also a promising method for efficient drug delivery into the eye. These MP-formulations exhibit a high drug-loading capacity, thermodynamic stability, and high versatility for loading both hydrophilic and hydrophobic drugs (Figure 9A) [208]. Molecular imprinting is another technique used for the development a drug template on a flexible network of macromolecules (e.g. polymers) based on a lock and key concept. According to this theory, only the enzymes/molecules (keys) with particular active binding sites and unique 
geometrical structures are suitable for a specific substrate (locks). Therefore, molecules with incorrect shape or functionality cannot be recognized to by the substrate [204]. This technique provides higher drug affinity (generally through hydrogen binding, ionic or hydrophobic interactions) and improves the uptake and sustained release of the drug [204]. Based on these technique, one or more functional monomers is polymerized in the presence of a drug template (Figure 9B). The functional monomers need to possess some characteristics such as chemical compatibility with the lens composition and high affinity for the drug template. Acrylic acid, methacrylic acid, acrylamide, methyl methacrylate, hydroxyethyl methacrylate and $\mathrm{N}$ vinylpyrrolidone are the most widely used functional monomers for ophthalmic drug delivery in CLs [205]. 

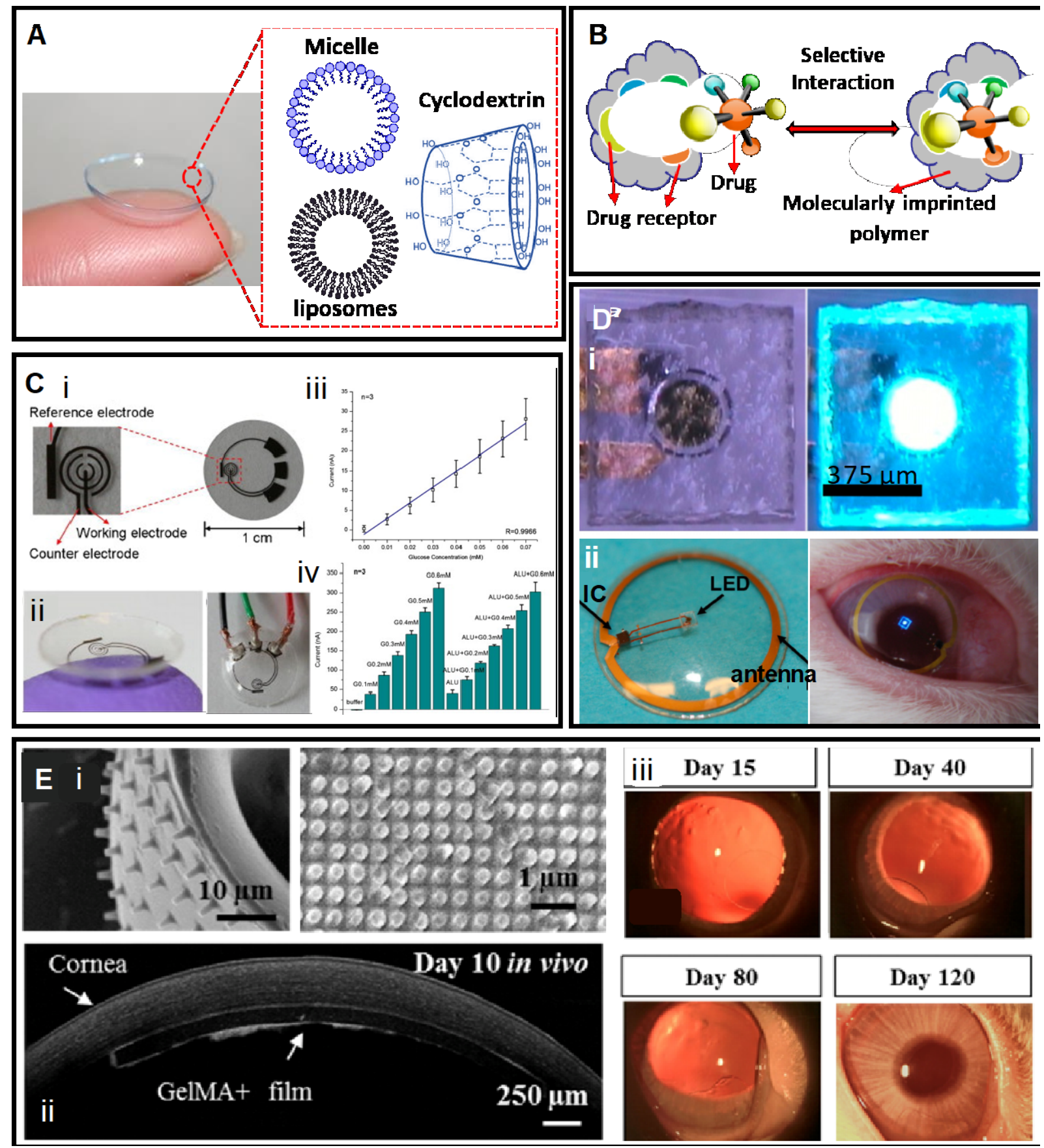

Figure 9. Examples of CLs and prefabricated patches. (A) Schematic of the NP-laden CLs, using nanostructures such as liposomes, cyclodextrins and micelles. (B) Schematic of the molecularly imprinted polymers used for highly selective drug delivery systems in CLs. (C) (i) Scheme and (ii) images of a CL with an integrated amperometric sensor for glucose monitoring and analysis. (iii) Linear correlation of current versus glucose concentrations $(0.01-0.07 \mathrm{mM}$ range) and, (iv) sensor accuracy and repeatability in the presence of interfering agents. Adapted from Yao et al. [226] 
with permission from Elsevier, copyright 2011. (D) LED display in a contact lens; (i) polyethylene terephthalate (PET) chip containing a LED with and without voltage application, (ii) contact lens device containing a LED, and anthena and a power harvesting system to display a single pixel wirelessly and its successful operation on a live rabbit eye. Adapted from Lingley et al., 2011 [227] with permission from IOP, copyright 2011. (E) (i) Representative images of nanopatterned adhesive and bioactive patch used for corneal tissue engineering applications. (ii) A optical coherence tomography (OCT)image of implanted nanopatterned patch in rabbit eye at day 10 of the study. (iii) Slit lamp images of the regeneration process of rabbit corneas at different time points after implantation of nanopatterned patch. Adapted from Rizwan et al. [228] with permission from Elsevier, copyright 2017.

Ocular diagnosis and biosensing is another promising application of CLs. For instance, Yao et al. designed a CL with an integrated amperometric sensor in order to monitor and analyze the glucose composition of tears of diabetic patients (Figure 9Ci-ii) [226]. The engineered biosensor showed a minimum detection capability of less than $0.01 \mathrm{mM}$ glucose, a high linearity in the normal range of tear glucose concentration $(0.1-0.6 \mathrm{mM})$, as well as high selectivity in the presence of interfering agents (Figure 9Ciii-iv) [226, 229]. Chen et al. reported a sensor to measure IOP intended to monitor glaucoma [230]. This sensor was integrated to a silicon CL and responded to changes in the eye curvature with high sensitivity ( $>200 \mathrm{ppm} / \mathrm{mmHg}$ of IOP in exvivo porcine eyes) and linearity in real time. Recently, CLs have been integrated into sensors for different molecules such as ions, proteins, urea, pyruvate, dopamine, ascorbate and lactate [199]. This is likely due to new technologies and developments in electronics, micro/nanofabrication techniques, biomaterials and biosensors [199]. Future advances along this line could enable the diagnosis and even treatment of different diseases such as Meibomian gland disease, AIDS, diabetes, cancer, glaucoma, ocular chlamydia trachomatis and keratoconus [199].

Advances in telecommunications are also impacting CL technology. For instance, Lingley et al. reported a design of a CL that was able to display a single pixel wirelessly [227]. They 
assembled a chip containing an Indium gallium nitride/ Gallium nitride (InGaN/GaN) LED onto a polyethylene terephthalate (PET) sheet emitting light when voltage was applied (Figure 9Di). The integration of an antenna, a wireless power harvesting system, the LED, and the polymeric substrate resulted in a CL capable of displaying a single pixel. The CL was operable and controllable remotely on live rabbit eyes (Figure Dii). These technological advances aim to develop a new generation of wearable CLs able to display multipixel images and interactive information.

In addition to bandage CLs, prefabricated patches have been extensively used for ocular tissue regeneration. A group of these prefabricated patches are inspired by the gecko's foot-hair as a successful strategy to enhance the adhesion of the patch to both wet and dry surfaces [231]. Using this technique, Rizwan et al. engineered a nano-patterned and bioactive patch that was used as an implantable cell-carrier construct for corneal repair [228] (Figure 9Ei). The engineered patch exhibited high adhesion strength and cell/patch interaction. In addition, it induced corneal defect regeneration, suggesting its potential as a micro/nanoengineered patch for corneal endothelial dysfunction repair (Figure 9Eii-iii) [228]. Vitrigel membranes are another group of prefabricated patches (described in section 3.1.3) [163, 232]. Chae et al. developed a collagen vitrigel membrane that could help reconstruction of the corneal epithelial layer and prevent epithelial hypertrophy, minimizing complications such as immune rejection or delay in epithelialization [163]. Due to the high transparency, low thickness, and high biocompatibility of the prefabricated membranes, they are promising biomaterials for corneal grafting as well as good alternative for corneal regeneration [163].

In the years to come, many more applications are expected to expand the physicians' resources to attend to patient needs after ocular surgery or injury. For example, the use of smart 
CLs could be extended to monitor the evolution of the ocular healing process, and to deliver, ondemand, the proper sequence of chemical and physical cues to favor tissue repair.

\section{Decellularized Corneas}

Despite the numerous advances in biomaterials science discussed above, biomaterial-based engineered corneas often do not faithfully replicate the microenvironment and microarchitecture of the cornea. Consequently, many efforts to repair corneal blindness have remained focused on corneal transplantation. Traditionally, corneal transplantations have relied on allogeneic donors. However, alternative approaches have been investigated as there is an insurmountable donor shortage. The transplantation of porcine corneas has particularly gained attention due to their availability, low cost, and comparable refractive and dimensional nature. However, porcine corneas are inherently xenogeneic, and decellularization is required prior to medical use.

Numerous decellularization techniques including chemical, physical, and enzymatic approaches have been developed over the years. Chemical approaches such as ionic detergents (e.g. sodium dodecyl sulfate) have been used on full-thickness porcine corneas to solubilize cellular membranes and remove $\alpha-G a l$ in, while leaving the cornea's architecture and structural protein such as collagens, laminins, and fibronectins largely intact [233]. Although anionic detergents such as Triton X-100 and zwitterionic detergents such as CHAPS have the capability to dissolve cell membranes while keeping the integrity of the extracellular matrix, they are reported to be less effective for corneal decellularization than ionic detergents (which tend to denaturate proteins). Specifically, current protocols leave a significant fraction of cells alive or leave numerous cell fragments behind, which can induce an immune response [234]. Cell lysation using hypotonic or hypertonic treatments has been proven to be effective and maintain the cornea's 
structure, and though nuclear fragments are left behind, this can be remedied by the inclusion of nucleases [235].

Other chemical approaches include exposing corneas to chelating agents (e.g. ethylenediaminetetraacetic acid), alcohols (e.g. ethanol), bases (e.g. ammonium hydroxide), or acids (e.g. peracetic acid). Many studies have also explored combinations of decellularization techniques, in particular, chelating agents are often combined with enzymes, and detergents are often combined with physical methods (e.g. ultrahigh hydrostatic pressures). Physical decellularization of corneal tissues can be based on snap freezing and/or lyophilizing or applying hydrostatic pressure, both of which disrupt the cell membrane [236-238]. These techniques can induce swelling due to changes in the cornea's architecture (e.g. increasing its porosity), and loss of structural protein such as glycosaminoglycans [239]. Enzymatic decellularization can be achieved using dispases, serine proteases, nucleases, and phospholipases. Interestingly, human serum is known to contain numerous enzymes including nucleases, and has been shown to effectively decellularize corneas [240]. In addition to the mentioned techniques, corneas can be gamma-irradiated to extend their shelf-life and reduce the risk of disease transmission [241].

Despite the claimed efficiencies of the decellularization protocols, the prevention of swelling, maintenance of architecture, preservation of transparency, and prevention of the loss of matrix proteins have remained common challenges. Biomaterials have been used to mitigate these drawbacks. For example, the polysaccharide dextran has been used to prevent corneal swelling and changes in the cornea's ultrastructure [242]. In addition, decellularized tissue scaffolds have been combined with HA to replace the lost structural proteins and to more effectively retain growth factors of choice [243, 244]. Although several studies have demonstrated that this approach holds great promise for treatments based on decellularized skin, efforts to determine the clinical potential 
of combinations of decellularized corneas and biomaterials have lagged behind. In addition, combining corneas with adhesive hydrogels could potentially increase successful grafting, drive suture-less applications, and steer encapsulated donor cells or recruited host cells.

\section{Conclusion and outlook}

The structural uniqueness of the human eye which enables vision is the driving force that pushes the scientific community to seek novel solutions that replace conventional surgical approaches to managing serious ocular injury and disease. Numerous efforts have been devoted to

preparing synthetic-based and naturally derived adhesives (e.g. cyanoacrylates, PEG based adhesives, protein- and polysaccharide-based adhesives), which address some of the risks and shortcomings associated with the application of sutures (Table 1). Owing to their distinct properties, each of these adhesives shows promise for use in different clinical settings, where retention, adhesiveness, or mechanical supports are required. The state-of-the-art in ocular wound care has as a result evolved considerably through the years from conventional suturing to the use of rationally designed biomaterials. Cyanoacrylates, often used as off-label adhesives for ocular wound closure, will be substantially improved or replaced for other 'eye-friendly' options such as new natural or synthetic polymeric materials.

In the near future, a new generation of ocular adhesives with tunable properties that not only fulfill the required design criteria, but also actively favor tissue repair will reach the market place. We can envision, for example, ocular scaffolds holding stem cells that can undergo differentiation and proliferation, or smart CLs that will monitor and speed up the wound healing process through on-line sensing and on-demand drug delivery. These and other examples of the integration of 
biomaterial engineering, electronics, micro- and nanotechnologies are expected to improve the healing process and will embody a new stage in ocular medicine with improved patient outcome.

\section{Acknowledgments}

N.A. acknowledges the support from the American Heart Association (AHA, 16SDG31280010) and National Institutes of Health (NIH) (R01EB023052; R01HL140618). GTdS gratefully acknowledge the funding received by CONACyT (scholarship 234713) and Fundación México in Harvard in the form of postdoctoral scholarships. MMA gratefully acknowledge the funding received from Tec de Monterrey and MIT Nanotechnology Program, the MIT International Science and Technology Initiatives (MISTI), and funding provided by CONACyT (Consejo Nacional de Ciencia y Tecnología, México) in the form of a sabbatical scholarship (262130). JL acknowledges financial support from Innovative Research Incentives Scheme Veni \#14328 of the Netherlands Organization for Scientific Research (NWO). RD acknowledges supports from Department of Defense (DoD) (W81XWH-18-1-0654) and Research to Prevent Blindness (RPB) Stein Innovation Award. AK acknowledges funding from the National Science Foundation (EFRI-1240443), IMMODGEL (602694), and the National Institutes of Health (EB012597, AR057837, DE021468, HL099073, AI105024, AR063745). 


\section{References}

[1] J.F. Acheson, C.J. Lyons, Ocular Morbidity Due to Monofilament Nylon Corneal Sutures, Eye 5 (1991) 7.

[2] M.W. Grinstaff, Designing hydrogel adhesives for corneal wound repair, Biomaterials 28(35) (2007) 5205-14.

[3] T. Oshika, G. Sugita, K. Miyata, T. Tokunaga, T. Samejima, C. Okamoto, Y. Ishii, Influence of tilt and decentration of scleral-sutured intraocular lens on ocular higher-order wavefront aberration, British Journal of Ophthalmology

91(2) (2007) 4.

[4] S.S. Bhatia, Ocular surface sealants and adhesives, Ocul Surf 4(3) (2006) 146-54.

[5] S.M. Chan, H.l.n. Boisjoly, Advances in the use of adhesives in ophthalmology, Current Opinion in Ophthalmology 15(4) (2004) 305-310.

[6] M.F. Refojo, C.H. Dohlman, B. Ahmad, J.M. Carroll, J.C. Allen, Evaluation of Adhesives for Corneal Surgery. , Archives of ophthalmology 80(5) (1968) 12.

[7] R.G. Webster, H.H. Slansky, M.F. Refojo, S.A. Boruchoff, C. Dohlman, The Use of Adhesive for the Closure of Corneal Perforations. Report of Two Cases, Arch Ophthalmol. 80(6) (1968) 5.

[8] D. Miki, K. Dastgheib, T. Kim, A. Pfister-Serres, K.A. Smeds, M. Inoue, D.L. Hatchell, M.W. Grinstaff, A Photopolymerized Sealant for Corneal Lacerations, Cornea 21(4) (2002) 393-399.

[9] J.J. Chae, D.G. Mulreany, Q. Guo, Q. Lu, J.S. Choi, I. Strehin, F.A. Espinoza, O. Schein, M.M. Trexler, K.S. Bower, J.H. Elisseeff, Application of a Collagen-Based Membrane and Chondroitin Sulfate-Based Hydrogel Adhesive for the Potential Repair of Severe Ocular Surface Injuries, Military Medicine 179(6) (2014) 686-694.

[10] H.C. Park, R. Champakalakshmi, P.P. Panengad, M. Raghunath, J.S. Mehta, Tissue adhesives in ocular surgery, Expert review of ophthalmology (2014).

[11] M. Mehdizadeh, J. Yang, Design strategies and applications of tissue bioadhesives, Macromol Biosci 13(3) (2013) 271-88.

[12] S. Bloomfield, A.H. Barnert, P.D. Kanter, The Use of Eastman 910 Monomer as an Adhesive in Ocular Surgery, American Journal of Ophthalmology 55(4) (1963) 742-748.

[13] A.T. Trott, Cyanoacrylate tissue adhesives. An advance in wound care, Journal of the American Medical Association 277(19) (1997) 1559-60.

[14] J.L. Alio, E. Mulet, H.F. Sakla, F. Gobbi, Efficacy of synthetic and biological bioadhesives in scleral tunnel phacoemulsification in eyes with high myopia, J Cataract Refract Surg 24(7) (1998) 983-8.

[15] J.A. Price Jr, J.A.C. Wadsworth, Evaluation of an adhesive in cataract wound closure, American Journal of Ophthalmology 68(4) (1969) 663-668.

[16] S.W. Meskin, D.C. Ritterband, D.E. Shapiro, J. Kusmierczyk, S.S. Schneider, J.A. Seedor, R.S. Koplin, Liquid bandage (2-octyl cyanoacrylate) as a temporary wound barrier in clear corneal cataract surgery, Ophthalmology 112(11) (2005) 2015-21.

[17] G.Y.S. Leung, V. Peponis, E.D. Varnell, D.S.C. Lam, H.E. Kaufman, Preliminary in vitro evaluation of 2-octyl cyanoacrylate (Dermabond ${ }^{\circledR}$ ) to seal corneal incisions, Cornea 24(8) (2005) 998-999.

[18] T. Kim, B.V. Kharod, Tissue adhesives in corneal cataract incisions, Curr Opin Ophthalmol 18(1) (2007) 39-43.

[19] R.G. Webster, The Use of Adhesive for the Closure of Corneal Perforations, Archives of ophthalmology 80(6) (1968) 705. 
[20] J.L. Alió, M.E. Mulet, J.C. Garcia, Use of cyanoacrylate tissue adhesive in small-incision cataract surgery, Ophthalmic Surgery and Lasers 27(4) (1996) 270-274.

[21] T. Shigemitsu, Y. Majima, The utilization of a biological adhesive for wound treatment: comparison of suture, self-sealing sutureless and cyanoacrylate closure in the tensile strength test, Int Ophthalmol 20(6) (1996) 323-8.

[22] K.J. Awan, P.G. Spaeth, Use of isobutyl 2 cyanoacrylate tissue adhesive in the repair of conjunctival fistula in filtering procedures for glaucoma, Annals of Ophthalmology 6(8) (1974) 851-853.

[23] F.J. Grady, M. Forbes, Tissue adhesive for repair of conjunctival buttonhole in glaucoma surgery, Am J Ophthalmol 68(4) (1969) 656-8.

[24] A.H. Zalta, R.H. Wieder, Closure of leaking filtering blebs with cyanoacrylate tissue adhesive, Br J Ophthalmol 75(3) (1991) 170-3.

[25] S. Al-Shahwan, A.A. Al-Torbak, I. Al-Jadaan, M. Omran, D.P. Edward, Long-term follow up of surgical repair of late bleb leaks after glaucoma filtering surgery, J Glaucoma 15(5) (2006) 432-6.

[26] A.R. Haslinda, Y. Azhany, R. Noor-Khairul, E. Zunaina, A.T. Liza-Sharmini, Cyanoacrylate tissue glue for wound repair in early posttrabeculectomy conjunctival bleb leak: a case series, Int Med Case Rep J 8 (2015) 145-50.

[27] M.E. Loane, A. Galanopoulos, The surgical management of leaking filtering blebs, Curr Opin Ophthalmol 10(2) (1999) 121-5.

[28] M.E. Hartnett, T. Hirose, Cyanoacrylate glue in the repair of retinal detachment associated with posterior retinal breaks in infants and children, Retina 18(2) (1998) 125-9.

[29] K. Hotta, A. Hirakata, T. Hida, The management of retinal detachments associated with choroidal colobomas by vitrectomy with cyanoacrylate retinopexy, Jpn J Ophthalmol 42(4) (1998) 323-6.

[30] B.W. McCuen Ii, T. Hida, S.M. Sheta, R. Machemer, Transvitreal cyanoacrylate retinopexy in the management of complicated retinal detachment, Transactions of the American Ophthalmological Society 85 (1987) 195-207.

[31] S.M. Sheta, T. Hida, B.W. McCuen, 2nd, Cyanoacrylate tissue adhesive in the management of recurrent retinal detachment caused by macular hole, Am J Ophthalmol 109(1) (1990) 28-32.

[32] A. Ohrstrom, S. Stenkula, L. Berglin, P. Naeser, Scleral reinforcement by a teflon graft and a tissue adhesive, Acta Ophthalmol 66(6) (1988) 643-6.

[33] L.C.B. Ferraz, S.A. Schellini, S.L. Wludarski, C.R. Padovani, D. Selva, S. Mueller, Extraocular muscle fixation to porous polyethylene orbital implants using 2-octyl-cyanoacrylate, European Journal of Ophthalmology 19(4) (2009) 527-529.

[34] B.K. Gupta, D. Edward, M.T. Duffy, 2-Octyl cyanoacrylate tissue adhesive and muscle attachment to porous anophthalmic orbital implants, Ophthal Plast Reconstr Surg 17(4) (2001) 264-9.

[35] J. Murube, E. Murube, Treatment of dry eye by blocking the lacrimal canaliculi, Surv Ophthalmol 40(6) (1996) 463-80.

[36] J.T. Patten, Punctal occlusion with n-butyl cyanoacrylate tissue adhesive, Ophthalmic Surg 7(2) (1976) 24-6.

[37] D.A. Ellis, A. Shaikh, The ideal tissue adhesive in facial plastic and reconstructive surgery, J Otolaryngol 19(1) (1990) 68-72. 
[38] D. Greene, R.J. Koch, R.L. Goode, Efficacy of octyl-2-cyanoacrylate tissue glue in blepharoplasty. A prospective controlled study of wound-healing characteristics, Arch Facial Plast Surg 1(4) (1999) 292-6.

[39] L.F. Perin, A. Helene, Jr., M.F. Fraga, Sutureless closure of the upper eyelids in blepharoplasty: use of octyl-2-cyanoacrylate, Aesthet Surg J 29(2) (2009) 87-92.

[40] D. Trivedi, M. McCalla, Z. Squires, M. Parulekar, Use of cyanoacrylate glue for temporary tarsorrhaphy in children, Ophthal Plast Reconstr Surg 30(1) (2014) 60-3.

[41] E.D. Donnenfeld, H.D. Perry, D.B. Nelson, Cyanoacrylate temporary tarsorrhaphy in the management of corneal epithelial defects, Ophthalmic Surg 22(10) (1991) 591-3.

[42] A.B. Leahey, J.D. Gottsch, W.J. Stark, Clinical experience with N-butyl cyanoacrylate (Nexacryl) tissue adhesive, Ophthalmology 100(2) (1993) 173-80.

[43] S. Rubab, D. French, A.V. Levin, Glued patches for children resistant to amblyopia occlusion therapy, Archives of ophthalmology 126(1) (2008) 133-4.

[44] R.A. Khodak, Removal of non-magnetic foreign bodies from deep layers of the cornea using cyanoacrylate glue, Oftalmologicheskii Zhurnal (8) (1987) 500-501.

[45] J.J. Arentsen, P.R. Laibson, E.J. Cohen, Management of corneal descemetoceles and perforations, Ophthalmic Surg 16(1) (1985) 29-33.

[46] A.R. Gasset, H.E. Kaufman, Epikeratoprosthesis. Replacement of superficial cornea by methyl methacrylate, Am J Ophthalmol 66(4) (1968) 641-5.

[47] A.E. Ardis, Preparation of monomeric alkyl alpha-cyano-acrylates, Google Patents, 1949.

[48] C. Vauthier, C. Dubernet, E. Fattal, H. Pinto-Alphandary, P. Couvreur, Poly(alkylcyanoacrylates) as biodegradable materials for biomedical applications, Advanced Drug Delivery Reviews 55(4) (2003) 519-548.

[49] F. Leonard, R.K. Kulkarni, G. Brandes, J. Nelson, J.J. Cameron, Synthesis and degradation of poly(alkyl- $\alpha$-cyanoacrylates), Journal of Applied Polymer Science 10(2) (1966) 259-272.

[50] M. Rana, V. Savant, A brief review of techniques used to seal corneal perforation using cyanoacrylate tissue adhesive, Cont Lens Anterior Eye 36(4) (2013) 156-8.

[51] G. Ciapetti, S. Stea, E. Cenni, A. Sudanese, D. Marraro, A. Toni, A. Pizzoferrato, Cytotoxicity testing of cyanoacrylates using direct contact assay on cell cultures, Biomaterials 15(1) (1994) 6367.

[52] Y.C. Tseng, Y. Tabata, S.H. Hyon, Y. Ikada, In vitro toxicity test of 2-cyanoacrylate polymers by cell culture method, J Biomed Mater Res 24(10) (1990) 1355-67.

[53] W.-L. Chen, C.-T. Lin, C.-Y. Hsieh, I.-H. Tu, W.Y. Chen, F.-R. Hu, Comparison of the bacteriostatic effects, corneal cytotoxicity, and the ability to seal corneal incisions among three different tissue adhesives, Cornea 26(10) (2007) 1228-1234.

[54] P.A. Leggat, D.R. Smith, U. Kedjarune, Surgical applications of cyanoacrylate adhesives: a review of toxicity, ANZ J Surg 77(4) (2007) 209-13.

[55] M.F. Refojo, C.H. Dohlman, The tensile strength of adhesive joints between eye tissues and alloplastic materials, Am J Ophthalmol 68(2) (1969) 248-255.

[56] R.A. Eiferman, J.W. Snyder, Antibacterial effect of cyanoacrylate glue, Archives of ophthalmology 101(6) (1983) 958-60.

[57] R.P. de Almeida Manzano, S.C. Naufal, R.Y. Hida, L.O. Guarnieri, M.C. Nishiwaki-Dantas, Antibacterial analysis in vitro of ethyl-cyanoacrylate against ocular pathogens, Cornea 25(3) (2006) 350-1. 
[58] I.L. Romero, J.B. Malta, C.B. Silva, L.M. Mimica, K.H. Soong, R.Y. Hida, Antibacterial properties of cyanoacrylate tissue adhesive: Does the polymerization reaction play a role?, Indian J Ophthalmol 57(5) (2009) 341-4.

[59] Y.J. Lee, H.S. Son, G.B. Jung, J.H. Kim, S. Choi, G.J. Lee, H.K. Park, Enhanced biocompatibility and wound healing properties of biodegradable polymer-modified allyl 2cyanoacrylate tissue adhesive, Mater Sci Eng C Mater Biol Appl 51 (2015) 43-50.

[60] M.D. Bona, B.W. Arthur, Cyanoacrylate tissue adhesive on a polyglactin scaffold in strabismus surgery: a laboratory study, J AAPOS 18(1) (2014) 21-5.

[61] F. Scognamiglio, A. Travan, I. Rustighi, P. Tarchi, S. Palmisano, E. Marsich, M. Borgogna, I. Donati, N. de Manzini, S. Paoletti, Adhesive and sealant interfaces for general surgery applications, J Biomed Mater Res B Appl Biomater 104(3) (2016) 626-39.

[62] T.B. Cavanaugh, J.D. Gottsch, Infectious keratitis and cyanoacrylate adhesive, Am J Ophthalmol 111(4) (1991) 466-72.

[63] G.D. Markowitz, S.E. Orlin, W.C. Frayer, A.P. Andrews, R.B. Prince, Corneal endothelial polymerization of histoacryl adhesive: a report of a new intraocular complication, Ophthalmic Surg 26(3) (1995) 256-8.

[64] A.P. Ferry, A.H. Barnert, Granulomatous keratitis resulting from use of cyanoacrylate adhesive for closure of perforated corneal ulcer, American Journal of Ophthalmology 72(3) (1971) 538-541.

[65] I. Strempel, Complications by liquid plastics in ophthalmic surgery. Histopathologic study, Dev Ophthalmol 13 (1987) 137-46.

[66] A.B. Leahey, J.D. Gottsch, Symblepharon associated with cyanoacrylate tissue adhesive, Archives of ophthalmology 111(2) (1993) 168.

[67] J.E. Siegal, G.W. Zaidman, Surgical removal of cyanoacrylate adhesive after accidental instillation in the anterior chamber, Ophthalmic Surg 20(3) (1989) 179-81.

[68] H.E. Kaufman, M.S. Insler, H.A. Ibrahim-Elzembely, S.C. Kaufman, Human fibrin tissue adhesive for sutureless lamellar keratoplasty and scleral patch adhesion: a pilot study, Ophthalmology 110(11) (2003) 2168-72.

[69] T.J. Sanborn, P.B. Messersmith, A.E. Barron, In situ crosslinking of a biomimetic peptidePEG hydrogel via thermally triggered activation of factor XIII, Biomaterials 23(13) (2002) 27032710.

[70] C.S. Bahney, T.J. Lujan, C.W. Hsu, M. Bottlang, J.L. West, B. Johnstone, Visible light photoinitiation of mesenchymal stem cell-laden bioresponsive hydrogels, Eur Cell Mater 22 (2011) 43-55; discussion 55.

[71] D.S. Kohane, R. Langer, Polymeric biomaterials in tissue engineering, Pediatr Res 63(5) (2008) 487-91.

[72] B. Yanez-Soto, S.J. Liliensiek, C.J. Murphy, P.F. Nealey, Biochemically and topographically engineered poly(ethylene glycol) diacrylate hydrogels with biomimetic characteristics as substrates for human corneal epithelial cells, J Biomed Mater Res A 101(4) (2013) 1184-94.

[73] S. Masket, J.A. Hovanesian, J. Levenson, F. Tyson, W. Flynn, M. Endl, P.A. Majmudar, S. Modi, R. Chu, M.B. Raizman, S.S. Lane, T. Kim, Hydrogel sealant versus sutures to prevent fluid egress after cataract surgery, J Cataract Refract Surg 40(12) (2014) 2057-66.

[74] D. Calladine, M. Ward, R. Packard, Adherent ocular bandage for clear corneal incisions used in cataract surgery, J Cataract Refract Surg 36(11) (2010) 1839-48.

[75] S.S. Ramsook, P.S. Hersh, Use of a hydrogel sealant in epithelial ingrowth removal after laser in situ keratomileusis, J Cataract Refract Surg 41(12) (2015) 2768-71. 
[76] FDA, RESURE SEALANT: Summary Of Safety And Effectiveness, 2014.

[77] S.J. Bryant, C.R. Nuttelman, K.S. Anseth, Cytocompatibility of UV and visible light photoinitiating systems on cultured NIH/3T3 fibroblasts in vitro, Journal of Biomaterials Science, Polymer Edition 11(5) (2000) 439-457.

[78] M.A. Carnahan, C. Middleton, J. Kim, T. Kim, M.W. Grinstaff, Hybrid dendritic-linear polyester-ethers for in situ photopolymerization, Journal of the American Chemical Society 124(19) (2002) 5291-3.

[79] J.P. Mazzoccoli, D.L. Feke, H. Baskaran, P.N. Pintauro, Mechanical and cell viability properties of crosslinked low- and high-molecular weight poly(ethylene glycol) diacrylate blends, J Biomed Mater Res A 93(2) (2010) 558-66.

[80] C. Alvarez-Lorenzo, F. Yanez, R. Barreiro-Iglesias, A. Concheiro, Imprinted soft contact lenses as norfloxacin delivery systems, Journal of Controlled Release 113(3) (2006) 236-244.

[81] S. Hoshi, F. Okamoto, M. Arai, T. Hirose, Y. Sugiura, Y. Kaji, T. Oshika, In Vivo and In Vitro Feasibility Studies of Intraocular Use of Polyethylene Glycol-Based Synthetic Sealant to Close Retinal Breaks in Porcine and Rabbit Eyes, Invest Ophthalmol Vis Sci 56(8) (2015) 470511.

[82] S. Lin-Gibson, S. Bencherif, J.A. Cooper, S.J. Wetzel, J.M. Antonucci, B.M. Vogel, F. Horkay, N.R. Washburn, Synthesis and characterization of PEG dimethacrylates and their hydrogels, Biomacromolecules 5(4) (2004) 1280-7.

[83] E. Margalit, G.Y. Fujii, J.C. Lai, P. Gupta, S.J. Chen, J.S. Shyu, D.V. Piyathaisere, J.D. Weiland, E. De Juan Jr, M.S. Humayun, Bioadhesives for intraocular use, Retina 20(5) (2000) 469-477.

[84] D. Kalayci, T. Fukuchi, P.G. Edelman, A.S. Sawhney, M.C. Mehta, T. Hirose, Hydrogel tissue adhesive for sealing corneal incisions, Ophthalmic Res 35(3) (2003) 173-6.

[85] L. Hartmann, K. Watanabe, L.L. Zheng, C.Y. Kim, S.E. Beck, P. Huie, J. Noolandi, J.R. Cochran, C.N. Ta, C.W. Frank, Toward the development of an artificial cornea: improved stability of interpenetrating polymer networks, J Biomed Mater Res B Appl Biomater 98(1) (2011) 8-17.

[86] B.I. Voit, A. Lederer, Hyperbranched and highly branched polymer architectures--synthetic strategies and major characterization aspects, Chemical reviews 109(11) (2009) 5924-73.

[87] C. Gao, D. Yan, Hyperbranched polymers: from synthesis to applications, Progress in Polymer Science 29(3) (2004) 183-275.

[88] A.M. Caminade, S. Fruchon, C.O. Turrin, M. Poupot, A. Ouali, A. Maraval, M. Garzoni, M. Maly, V. Furer, V. Kovalenko, J.P. Majoral, G.M. Pavan, R. Poupot, The key role of the scaffold on the efficiency of dendrimer nanodrugs, Nat Commun 6 (2015) 7722.

[89] S.M. Grayson, J.M. Frechet, Convergent dendrons and dendrimers: from synthesis to applications, Chemical reviews 101(12) (2001) 3819-68.

[90] J.A. Valencia-Gallegos, M.M. Álvarez, V.J. Martínez-Merino, High loaded dendrimers with free peripheral groups. , Tetrahedron Letters 56(48) (2015) 4.

[91] M. Wathier, P.J. Jung, M.A. Carnahan, T. Kim, M.W. Grinstaff, Dendritic macromers as in situ polymerizing biomaterials for securing cataract incisions, Journal of the American Chemical Society 126(40) (2004) 12744-12745.

[92] M. Fischer, F. Vögtle, Dendrimers: From Design to Application-A Progress Report, Angewandte Chemie International Edition 38(7) (1999) 884-905.

[93] J.M. Frechet, Dendrimers and supramolecular chemistry, Proc Natl Acad Sci USA 99(8) (2002) 4782-7. 
[94] S. Andre, P.J. Ortega, M.A. Perez, R. Roy, H.J. Gabius, Lactose-containing starburst dendrimers: influence of dendrimer generation and binding-site orientation of receptors (plant/animal lectins and immunoglobulins) on binding properties, Glycobiology 9(11) (1999) 1253-61.

[95] M.A. Carnahan, M.W. Grinstaff, Synthesis and Characterization of Poly(glycerol-succinic acid) Dendrimers, Macromolecules 34(22) (2001) 7648-7655.

[96] H. Namazi, M. Adeli, Dendrimers of citric acid and poly (ethylene glycol) as the new drugdelivery agents, Biomaterials 26(10) (2005) 1175-83.

[97] X. Duan, H. Sheardown, Dendrimer crosslinked collagen as a corneal tissue engineering scaffold: mechanical properties and corneal epithelial cell interactions., Biomaterials 27(26) (2006) 10.

[98] A.J. Velazquez, M.A. Carnahan, J. Kristinsson, S. Stinnett, M.W. Grinstaff, T. Kim, New dendritic adhesives for sutureless ophthalmic surgical procedures: in vitro studies of corneal laceration repair, Archives of ophthalmology 122(6) (2004) 867-70.

[99] M.W. Grinstaff, Biodendrimers: new polymeric biomaterials for tissue engineering, Chemistry - A European Journal 8(13) (2002) 2839-46.

[100] P.C. Kang, M.A. Carnahan, M. Wathier, M.W. Grinstaff, T. Kim, Novel tissue adhesives to secure laser in situ keratomileusis flaps, J Cataract Refract Surg 31(6) (2005) 1208-12.

[101] M. Wathier, C.S. Johnson, T. Kim, M.W. Grinstaff, Hydrogels formed by multiple peptide ligation reactions to fasten corneal transplants, Bioconjug Chem 17(4) (2006) 873-6.

[102] A.M. Oelker, J.A. Berlin, M. Wathier, M.W. Grinstaff, Synthesis and Characterization of Dendron Cross-Linked PEG Hydrogels as Corneal Adhesives, Biomacromolecules 12(5) (2011) 1658-1665.

[103] M.B. Applegate, B.P. Partlow, J. Coburn, B. Marelli, C. Pirie, R. Pineda, D.L. Kaplan, F.G. Omenetto, Photocrosslinking of Silk Fibroin Using Riboflavin for Ocular Prostheses, Adv Mater 28(12) (2016) 2417-20.

[104] M. Radosevich, H. Goubran, T. Burnouf, Fibrin sealant: scientific rationale, production methods, properties, and current clinical use, Vox sanguinis 72(3) (1997) 133-143.

[105] A. Bochyńska, G. Hannink, D. Grijpma, P. Buma, Tissue adhesives for meniscus tear repair: an overview of current advances and prospects for future clinical solutions. 27(5) (2016) 1-18.

[106] R. Ariëns, Novel mechanisms that regulate clot structure/function, Thrombosis research 141 (2016) S25-S27.

[107] R. Doolittle, The Conversion of Fibrinogen to Fibrin: A Brief History of Some Key Events, Matrix Biology In Press (2016).

[108] M.M. Domingues, F.L. Macrae, C. Duval, H.R. McPherson, K.I. Bridge, R.A. Ajjan, V.C. Ridger, S.D. Connell, H. Philippou, R.A. Ariens, Thrombin and fibrinogen gamma' impact clot structure by marked effects on intrafibrillar structure and protofibril packing, Blood 127(4) (2016) 487-95.

[109] M.W. Mosesson, Fibrinogen and fibrin structure and functions, J Thromb Haemost 3(8) (2005) 1894-904.

[110] A.S. Wolberg, Thrombin generation and fibrin clot structure, Blood Rev 21(3) (2007) 13142.

[111] D.M. Harris, K.H. Siedentop, K.R. Ham, B. Sanchez, Autologous fibrin tissue adhesive biodegration and systemic effects, Laryngoscope 97(10) (1987) 1141-4.

[112] D.H. Sierra, D.S. Feldman, R. Saltz, S. Huang, A method to determine shear adhesive strength of fibrin sealants, J Appl Biomater 3(2) (1992) 147-51. 
[113] A. Sharma, R. Kaur, S. Kumar, P. Gupta, S. Pandav, B. Patnaik, A. Gupta, Fibrin glue versus N-butyl-2-cyanoacrylate in corneal perforations, Ophthalmology 110(2) (2003) 291-8.

[114] I. Bahar, D. Weinberger, M. Lusky, R. Avisar, A. Robinson, D. Gaton, Fibrin glue as a suture substitute: histological evaluation of trabeculectomy in rabbit eyes, Current eye research 31(1) (2006) 31-36.

[115] S. Srinivasan, M. Dollin, P. McAllum, Y. Berger, D.S. Rootman, A.R. Slomovic, Fibrin glue versus sutures for attaching the conjunctival autograft in pterygium surgery: a prospective observer masked clinical trial, Br J Ophthalmol 93(2) (2009) 215-8.

[116] Y. Rizvi, P. Rai, A. Dokania, Clinical outcome of Conjunctival auto-grafting in Pterygium surgery using Fibrin glue and sutures, Indian Journal of Basic and Applied Medical Research 4(2) (2015) 587-594.

[117] J. Marticorena, M. Rodríguez-Ares, R. Touriño, P. Mera, M. Valladares, J. Martinez-de-laCasa, J. Benitez-del-Castillo, Pterygium surgery: conjunctival autograft using a fibrin adhesive, Cornea 25(1) (2006) 34-36.

[118] A.X. Zhou, B.K. Ambati, Sutureless Lamellar Corneoscleral Patch Graft With Fibrin Sealant for Limbal Dermoid Removal, J Pediatr Ophthalmol Strabismus 53 Online (2016) e22-5.

[119] B. Duchesne, H. Tahi, A. Galand, Use of human fibrin glue and amniotic membrane transplant in corneal perforation, Cornea 20(2) (2001) 230-232.

[120] P. Rama, S. Bonini, A. Lambiase, O. Golisano, P. Paterna, M. De Luca, G. Pellegrini, Autologous fibrin-cultured limbal stem cells permanently restore the corneal surface of patients with total limbal stem cell deficiency1, Transplantation 72(9) (2001) 1478-1485.

[121] B. Han, I.R. Schwab, T.K. Madsen, R.R. Isseroff, A fibrin-based bioengineered ocular surface with human corneal epithelial stem cells, Cornea 21(5) (2002) 505-10.

[122] B. Sonmez, U. Beden, Fibrin glue-assisted sutureless limbal stem cell transplantation surgery for the treatment of severe ocular chemical injury, Cornea 30(3) (2011) 296-300.

[123] A. Queiroz de Paiva, L. Abreu de Azevedo Fraga, V. Torres, Surgical Reconstruction of Ocular Surface Tumors Using Fibrin Sealant Tissue Adhesive, Ocular Oncology and Pathology 2(4) (2016) 207-211.

[124] M. Bungum, P. Humaidan, L. Bungum, Recombinant human albumin as protein source in culture media used for IVF: a prospective randomized study, Reproductive biomedicine online 4(3) (2002) 233-236.

[125] S. Sugio, A. Kashima, S. Mochizuki, M. Noda, K. Kobayashi, Crystal structure of human serum albumin at $2.5 \AA$ resolution, Protein engineering 12(6) (1999) 439-446.

[126] H.H. Chao, D.F. Torchiana, BioGlue: albumin/glutaraldehyde sealant in cardiac surgery, J Card Surg 18(6) (2003) 500-3.

[127] W. Fürst, A. Banerjee, Release of glutaraldehyde from an albumin-glutaraldehyde tissue adhesive causes significant in vitro and in vivo toxicity, The Annals of thoracic surgery 79(5) (2005) 1522-1528.

[128] K. Kobayashi, Summary of recombinant human serum albumin development, Biologicals 34(1) (2006) 55-9.

[129] H. Cao, L. Sun, L. Liu, J. Li, L. Tang, Y. Guo, Q. Mei, J. He, D. Yin, Structural consistency analysis of recombinant and wild-type human serum albumin Journal of Molecular Structure 1127 (2017) 1-5.

[130] J.J. Khadem, M.R. Dana, Photodynamic biologic tissue glue in perforating rabbit corneal wounds, J Clin Laser Med Surg 18(3) (2000) 125-9. 
[131] J. Khadem, M. Martino, F. Anatelli, M.R. Dana, M.R. Hamblin, Healing of perforating rat corneal incisions closed with photodynamic laser-activated tissue glue, Lasers Surg Med 35(4) (2004) 304-11.

[132] K. Tal, E. Strassmann, N. Loya, A. Ravid, N. Kariv, D. Weinberger, A. Katzir, D. Gaton, Corneal cut closure using temperature-controlled CO2 laser soldering system, Lasers in medical science 30(4) (2015) 1367-1371.

[133] V.L. Stefanelli, T.H. Barker, The evolution of fibrin-specific targeting strategies, Journal of Materials Chemistry B 3(7) (2015) 1177-1186.

[134] I. Rimshan, D. Ryabkin, M. Savelyev, N. Zhurbina, I. Pyanov, E. Eganova, A. Pavlov, V. Podgaetsky, L. Ichkitidze, S. Selishchev, The tensile strength characteristics study of the laser welds of biological tissue using the nanocomposite solder, Saratov Fall Meeting 2015, International Society for Optics and Photonics, Saratov, Russian Federation 2016, pp. 99170I99170I-7.

[135] A.M. Gobin, D. O'Neal, N.J. Halas, R.A. Drezek, J.L. West, Laser tissue soldering with near-infrared absorbing nanoparticles, Biomedical Optics 2005, International Society for Optics and Photonics, San Jose, CA, 2005, pp. 261-266.

[136] D.P. Poppas, S.M. Schlossberg, Laser tissue welding in urologic surgery, Urology 43(2) (1994) 143-8.

[137] C.M. Elvin, T. Vuocolo, A.G. Brownlee, L. Sando, M.G. Huson, N.E. Liyou, P.R. Stockwell, R.E. Lyons, M. Kim, G.A. Edwards, G. Johnson, G.A. McFarland, J.A. Ramshaw, J.A. Werkmeister, A highly elastic tissue sealant based on photopolymerised gelatin, Biomaterials 31(32) (2010) 8323-31.

[138] J. Bella, Collagen structure: new tricks from a very old dog, Biochemical Journal 473(8) (2016) 1001-1025.

[139] K.M. Meek, C. Boote, The organization of collagen in the corneal stroma, Exp Eye Res 78(3) (2004) 503-12.

[140] D.M. Maurice, The structure and transparency of the cornea, J Physiol 136(2) (1957) 26386.

[141] C. Baldock, C. Gilpin, A. Koster, U. Ziese, K. Kadler, C. Kielty, D. Holmes, Threedimensional reconstructions of extracellular matrix polymers using automated electron tomography, Journal of structural biology 138(1) (2002) 130-136.

[142] E. Spoerl, M. Huhle, T. Seiler, Induction of cross-links in corneal tissue, Exp Eye Res 66(1) (1998) 97-103.

[143] F. Raiskup-Wolf, A. Hoyer, E. Spoerl, L.E. Pillunat, Collagen crosslinking with riboflavin and ultraviolet-A light in keratoconus: long-term results, J Cataract Refract Surg 34(5) (2008) 796801.

[144] P. Matteini, F. Rossi, L. Menabuoni, R. Pini, Microscopic characterization of collagen modifications induced by low-temperature diode-laser welding of corneal tissue, Lasers Surg Med 39(7) (2007) 597-604.

[145] E. Coskunseven, M.R. Jankov, 2nd, F. Hafezi, Contralateral eye study of corneal collagen cross-linking with riboflavin and UVA irradiation in patients with keratoconus, J Refract Surg 25(4) (2009) 371-6.

[146] B. Wire, Avedro receives FDA approval for Photrexa ${ }^{\circledR}$ Viscous, Photrexa ${ }^{\circledR}$ and the KXL ${ }^{\circledR}$ System for corneal cross-linking <http://www.businesswire.com/news/home/20160418006610/en/Avedro-Receives-FDAApproval-Photrexa\%C2\%AE-Viscous-Photrexa\%C2\%AE>, 2016 (accessed June 28, 2016.2016). 
[147] O. Richoz, A. Hammer, D. Tabibian, Z. Gatzioufas, F. Hafezi, The biomechanical effect of corneal collagen cross-linking (CXL) with riboflavin and UV-A is oxygen dependent, Translational Vision Science \& Technology 2(7) (2013) 6.

[148] E.Y. Kim, N. Tripathy, S.A. Cho, D. Lee, G. Khang, Collagen type I-PLGA film as an efficient substratum for corneal endothelial cells regeneration, Journal of Tissue Engineering and Regenerative Medicine (2016) n/a-n/a.

[149] G. Noguera, W.S. Lee, J. Castro-Combs, R.S. Chuck, B. Soltz, R. Soltz, A. Behrens, Novel laser-activated solder for sealing corneal wounds, Invest Ophthalmol Vis Sci 48(3) (2007) 103842.

[150] X. Xiao, S. Pan, X. Liu, X. Zhu, C.J. Connon, J. Wu, S. Mi, In vivo study of the biocompatibility of a novel compressed collagen hydrogel scaffold for artificial corneas, J Biomed Mater Res A 102(6) (2014) 1782-7.

[151] X. Calderón-Colón, Z. Xia, J.L. Breidenich, D.G. Mulreany, Q. Guo, O.M. Uy, J.E. Tiffany, D.E. Freund, R.L. McCally, O.D. Schein, J.H. Elisseeff, M.M. Trexler, Structure and properties of collagen vitrigel membranes for ocular repair and regeneration applications, Biomaterials 33(33) (2012) 8286-8295.

[152] L. Zhao, X. Li, J. Zhao, S. Ma, X. Ma, D. Fan, C. Zhu, Y. Liu, A novel smart injectable hydrogel prepared by microbial transglutaminase and human-like collagen: Its characterization and biocompatibility, Mater Sci Eng C Mater Biol Appl 68 (2016) 317-26.

[153] H. Miyashita, S. Shimmura, H. Kobayashi, T. Taguchi, N. Asano-Kato, Y. Uchino, M. Kato, J. Shimazaki, J. Tanaka, K. Tsubota, Collagen-immobilized poly (vinyl alcohol) as an artificial cornea scaffold that supports a stratified corneal epithelium, Journal of Biomedical Materials Research Part B: Applied Biomaterials 76(1) (2006) 56-63.

[154] J. Zhang, A.M. Sisley, A.J. Anderson, A.J. Taberner, C.N. McGhee, D.V. Patel, Characterization of a Novel Collagen Scaffold for Corneal Tissue Engineering, Tissue Eng Part C Methods 22(2) (2015) 165-172.

[155] M. Rafat, F. Li, P. Fagerholm, N.S. Lagali, M.A. Watsky, R. Munger, T. Matsuura, M. Griffith, PEG-stabilized carbodiimide crosslinked collagen-chitosan hydrogels for corneal tissue engineering, Biomaterials 29(29) (2008) 3960-72.

[156] W. Liu, M. Griffith, F. Li, Alginate microsphere-collagen composite hydrogel for ocular drug delivery and implantation, J Mater Sci Mater Med 19(11) (2008) 3365-71.

[157] W. Liu, K. Merrett, M. Griffith, P. Fagerholm, S. Dravida, B. Heyne, J.C. Scaiano, M.A. Watsky, N. Shinozaki, N. Lagali, R. Munger, F. Li, Recombinant human collagen for tissue engineered corneal substitutes, Biomaterials 29(9) (2008) 1147-58.

[158] W. Liu, C. Deng, C.R. McLaughlin, P. Fagerholm, N.S. Lagali, B. Heyne, J.C. Scaiano, M.A. Watsky, Y. Kato, R. Munger, Collagen-phosphorylcholine interpenetrating network hydrogels as corneal substitutes, Biomaterials 30(8) (2009) 1551-1559.

[159] C. Deng, F. Li, J.M. Hackett, S.H. Chaudhry, F.N. Toll, B. Toye, W. Hodge, M. Griffith, Collagen and glycopolymer based hydrogel for potential corneal application, Acta Biomater 6(1) (2010) 187-94.

[160] J.-I. Ahn, L. Kuffova, K. Merrett, D. Mitra, J.V. Forrester, F. Li, M. Griffith, Crosslinked collagen hydrogels as corneal implants: effects of sterically bulky vs. non-bulky carbodiimides as crosslinkers, Acta biomaterialia 9(8) (2013) 7796-7805.

[161] W. McIntosh Ambrose, A. Salahuddin, S. So, S. Ng, S. Ponce Márquez, T. Takezawa, O. Schein, J. Elisseeff, Collagen vitrigel membranes for the in vitro reconstruction of separate corneal 
epithelial, stromal, and endothelial cell layers, Journal of Biomedical Materials Research Part B: Applied Biomaterials 90B(2) (2009) 818-831.

[162] Q. Guo, J.M. Phillip, S. Majumdar, P.H. Wu, J. Chen, X. Calderon-Colon, O. Schein, B.J. Smith, M.M. Trexler, D. Wirtz, J.H. Elisseeff, Modulation of keratocyte phenotype by collagen fibril nanoarchitecture in membranes for corneal repair, Biomaterials 34(37) (2013) 9365-72.

[163] J.J. Chae, W. McIntosh Ambrose, F.A. Espinoza, D.G. Mulreany, S. Ng, T. Takezawa, M.M. Trexler, O.D. Schein, R.S. Chuck, J.H. Elisseeff, Regeneration of corneal epithelium utilizing a collagen vitrigel membrane in rabbit models for corneal stromal wound and limbal stem cell deficiency, Acta Ophthalmologica 93(1) (2015) e57-e66.

[164] S. Yamamoto, A. Hirata, S. Ishikawa, K. Ohta, K.-i. Nakamura, S. Okinami, Feasibility of using gelatin-microbial transglutaminase complex to repair experimental retinal detachment in rabbit eyes, Graefe's Archive for Clinical and Experimental Ophthalmology 251(4) (2013) 11091114.

[165] M.K. McDermott, T. Chen, C.M. Williams, K.M. Markley, G.F. Payne, Mechanical properties of biomimetic tissue adhesive based on the microbial transglutaminase-catalyzed crosslinking of gelatin, Biomacromolecules 5(4) (2004) 1270-9.

[166] T. Chen, R. Janjua, M.K. McDermott, S.L. Bernstein, S.M. Steidl, G.F. Payne, Gelatin-based biomimetic tissue adhesive. Potential for retinal reattachment, J Biomed Mater Res B Appl Biomater 77(2) (2006) 416-22.

[167] I. Noshadi, S. Hong, K.E. Sullivan, E. Shirzaei Sani, R. Portillo-Lara, A. Tamayol, S.R. Shin, A.E. Gao, W.L. Stoppel, L.D. Black Iii, A. Khademhosseini, N. Annabi, In vitro and in vivo analysis of visible light crosslinkable gelatin methacryloyl (GelMA) hydrogels, Biomater Sci (2017).

[168] A. Assmann, A. Vegh, M. Ghasemi-Rad, S. Bagherifard, G. Cheng, E.S. Sani, G.U. RuizEsparza, I. Noshadi, A. Lassaletta, S. Gangadharan, A. Tamayol, A. Khademhosseini, N. Annabi, A highly adhesive and naturally derived sealant, Biomaterials 140 (2017) 115-127.

[169] N. Annabi, D. Rana, E. Shirzaei Sani, R. Portillo-Lara, J.L. Gifford, M.M. Fares, S.M. Mithieux, A.S. Weiss, Engineering a sprayable and elastic hydrogel adhesive with antimicrobial properties for wound healing, Biomaterials 139 (2017) 229-243.

[170] R. Watanabe, R. Hayashi, Y. Kimura, Y. Tanaka, T. Kageyama, S. Hara, Y. Tabata, K. Nishida, A novel gelatin hydrogel carrier sheet for corneal endothelial transplantation, Tissue engineering Part A 17(17-18) (2011) 2213-2219.

[171] J.-Y. Lai, Biocompatibility of chemically cross-linked gelatin hydrogels for ophthalmic use, Journal of Materials Science: Materials in Medicine 21(6) (2010) 1899-1911.

[172] Q. Li, C.G. Williams, D.D. Sun, J. Wang, K. Leong, J.H. Elisseeff, Photocrosslinkable polysaccharides based on chondroitin sulfate, J Biomed Mater Res A 68(1) (2004) 28-33.

[173] I. Strehin, W.M. Ambrose, O. Schein, A. Salahuddin, J. Elisseeff, Synthesis and characterization of a chondroitin sulfate-polyethylene glycol corneal adhesive, J Cataract Refract Surg 35(3) (2009) 567-76.

[174] J.M. Reyes, S. Herretes, A. Pirouzmanesh, D.A. Wang, J.H. Elisseeff, A. Jun, P.J. McDonnell, R.S. Chuck, A. Behrens, A modified chondroitin sulfate aldehyde adhesive for sealing corneal incisions, Invest Ophthalmol Vis Sci 46(4) (2005) 1247-50.

[175] D.A. Wang, S. Varghese, B. Sharma, I. Strehin, S. Fermanian, J. Gorham, D.H. Fairbrother, B. Cascio, J.H. Elisseeff, Multifunctional chondroitin sulphate for cartilage tissue-biomaterial integration, Nature materials 6(5) (2007) 385-92. 
[176] I. Strehin, Z. Nahas, K. Arora, T. Nguyen, J. Elisseeff, A versatile pH sensitive chondroitin sulfate-PEG tissue adhesive and hydrogel, Biomaterials 31(10) (2010) 2788-97.

[177] A. Pirouzmanesh, S. Herretes, J.M. Reyes, O. Suwan-apichon, R.S. Chuck, D.A. Wang, J.H. Elisseeff, W.J. Stark, A. Behrens, Modified microkeratome-assisted posterior lamellar keratoplasty using a tissue adhesive, Archives of ophthalmology 124(2) (2006) 210-4.

[178] C. Hiemstra, L.J. van der Aa, Z. Zhong, P.J. Dijkstra, J. Feijen, Novel in Situ Forming, Degradable Dextran Hydrogels by Michael Addition Chemistry: Synthesis, Rheology, and Degradation, Macromolecules 40(4) (2007) 1165-1173.

[179] R. Jin, C. Hiemstra, Z. Zhong, J. Feijen, Enzyme-mediated fast in situ formation of hydrogels from dextran-tyramine conjugates, Biomaterials 28(18) (2007) 2791-800.

[180] M. Araki, H. Tao, N. Nakajima, H. Sugai, T. Sato, S.H. Hyon, T. Nagayasu, T. Nakamura, Development of new biodegradable hydrogel glue for preventing alveolar air leakage, J Thorac Cardiovasc Surg 134(5) (2007) 1241-8.

[181] M. Araki, H. Tao, T. Sato, N. Nakajima, H. Sugai, S.H. Hyon, T. Nagayasu, T. Nakamura, Creation of a uniform pleural defect model for the study of lung sealants, J Thorac Cardiovasc Surg 134(1) (2007) 145-51.

[182] M. Takaoka, T. Nakamura, H. Sugai, A.J. Bentley, N. Nakajima, N.J. Fullwood, N. Yokoi, S.H. Hyon, S. Kinoshita, Sutureless amniotic membrane transplantation for ocular surface reconstruction with a chemically defined bioadhesive, Biomaterials 29(19) (2008) 2923-31.

[183] M. Takaoka, T. Nakamura, H. Sugai, A.J. Bentley, N. Nakajima, N. Yokoi, N.J. Fullwood, S.H. Hyon, S. Kinoshita, Novel sutureless keratoplasty with a chemically defined bioadhesive, Invest Ophthalmol Vis Sci 50(6) (2009) 2679-85.

[184] S.K. Bhatia, S.D. Arthur, H.K. Chenault, G.K. Kodokian, Interactions of polysaccharidebased tissue adhesives with clinically relevant fibroblast and macrophage cell lines, Biotechnol Lett 29(11) (2007) 1645-9.

[185] S.K. Bhatia, S.D. Arthur, H.K. Chenault, G.D. Figuly, G.K. Kodokian, Polysaccharide-based tissue adhesives for sealing corneal incisions, Curr Eye Res 32(12) (2007) 1045-50.

[186] H.K. Chenault, S.K. Bhatia, W.G. Dimaio, G.L. Vincent, W. Camacho, A. Behrens, Sealing and healing of clear corneal incisions with an improved dextran aldehyde-PEG amine tissue adhesive, Curr Eye Res 36(11) (2011) 997-1004.

[187] S.J.T. Lerit, J.M.R. Abaño, Comparison of Tensile Strength of Fibrin Glue,- 2-Octyl Cyanoacrylate,- Liquid Ocular Bandage,- and Conventional Nylon 10-0 Sutures in Corneal Laceration Repair in an Animal Model, Philippine Academy of Ophthalmology 37 (2012) 52-58. [188] C.S. Johnson, M. Wathier, M. Grinstaff, T. Kim, In vitro sealing of clear corneal cataract incisions with a novel biodendrimer adhesive, Archives of ophthalmology 127(4) (2009) 430-4.

[189] A. Patel, K. Cholkar, V. Agrahari, A.K. Mitra, Ocular drug delivery systems: An overview, World J Pharmacol 2(2) (2013) 47-64.

[190] M.C. Giano, Z. Ibrahim, S.H. Medina, K.A. Sarhane, J.M. Christensen, Y. Yamada, G. Brandacher, J.P. Schneider, Injectable bioadhesive hydrogels with innate antibacterial properties, Nat Commun 5 (2014) 4095.

[191] J.R.R. Ho-Wah Hui, Ocular delivery of progesterone using a bioadhesive polymer Int J Pharmaceut (1985).

[192] G.S. William Yee, Benjamin Hawkins, Characterization of silver nanoparticle-infused tissue adhesive for ophthalmic use, Journal of the mechanical behavior of biomedical materials 55 (2016). 
[193] A.-E.H.A.-E. Mohammed Mostafa Ibrahim, Osama Abd-Elazeem Soliman, Monica M. Jablonski, Natural bioadhesive biodegradable nanoparticles-based topical ophthalmic formulations for sustained celecoxib release: In vitro study, Transl Vis Sci Technol (2015).

[194] Y. Zhang, J. Zhang, M. Chen, H. Gong, S. Thamphiwatana, L. Eckmann, W. Gao, L. Zhang, A Bioadhesive Nanoparticle-Hydrogel Hybrid System for Localized Antimicrobial Drug Delivery, ACS Appl Mater Interfaces 8(28) (2016) 18367-74.

[195] H. Song, Y. Ahmad Nor, M. Yu, Y. Yang, J. Zhang, H. Zhang, C. Xu, N. Mitter, C. Yu, Silica Nanopollens Enhance Adhesion for Long-Term Bacterial Inhibition, Journal of the American Chemical Society 138(20) (2016) 6455-62.

[196] S.C. Sanjay K. Motwani, Sushma Talegaonkar, Kanchan Kohli, Farhan J. Ahmad, Roop K. Khar, Chitosan-sodium alginate nanoparticles as submicroscopic reservoirs for ocular delivery: formulation, optimisation and in vitro characterisation., Eur J Pharm Biopharm (2008).

[197] D. Sensoy, E. Cevher, A. Sarici, M. Yilmaz, A. Ozdamar, N. Bergisadi, Bioadhesive sulfacetamide sodium microspheres: evaluation of their effectiveness in the treatment of bacterial keratitis caused by Staphylococcus aureus and Pseudomonas aeruginosa in a rabbit model, Eur J Pharm Biopharm 72(3) (2009) 487-95.

[198] V. Baeyens, O. Felt-Baeyens, S. Rougier, S. Pheulpin, B. Boisrame, R. Gurny, Clinical evaluation of bioadhesive ophthalmic drug inserts (BODI) for the treatment of external ocular infections in dogs, J Control Release 85(1-3) (2002) 163-8.

[199] N.M. Farandos, A.K. Yetisen, M.J. Monteiro, C.R. Lowe, S.H. Yun, Contact lens sensors in ocular diagnostics, Advanced healthcare materials 4(6) (2015) 792-810.

[200] M. Mohammadpour, A. Amouzegar, H. Hashemi, M. Jabbarvand, H. Kordbacheh, F. Rahimi, M.N. Hashemian, Comparison of Lotrafilcon B and Balafilcon A silicone hydrogel bandage contact lenses in reducing pain and discomfort after photorefractive keratectomy: A contralateral eye study, Cont Lens Anterior Eye 38(3) (2015) 211-4.

[201] R. Gil-Cazorla, M.A. Teus, J.L. Hernández-Verdejo, L. De Benito-Llopis, M. GarcíaGonzález, Comparative study of two silicone hydrogel contact lenses used as bandage contact lenses after LASEK, Optometry \& Vision Science 85(9) (2008) 884-888.

[202] M.A. Grentzelos, S. Plainis, N.I. Astyrakakis, V.F. Diakonis, G.D. Kymionis, P. Kallinikos, I.G. Pallikaris, Efficacy of 2 types of silicone hydrogel bandage contact lenses after photorefractive keratectomy. , Journal of Cataract \& Refractive Surgery 35(12) (2009) 2103-2108.

[203] B. Chou, The Evolution of Silicone Hydrogel Lenses. The chemistry and material characteristics of current disposable silicone hydrogels in the United States, Contact Lens Spectrum (June ) (2008).

[204] H.P. Filipe, J. Henriques, P. Reis, P.C. Silva, M.J. Quadrado, A.P. Serro, Contact lenses as drug controlled release systems: a narrative review, Revista Brasileira de Oftalmologia 75(3) (2016) 241-247.

[205] K.-H. Hsu, S. Gause, A. Chauhan, Review of ophthalmic drug delivery by contact lenses, Journal of Drug Delivery Science and Technology 24(2) (2014) 123-135.

[206] A.G. Gallagher, J.A. Alorabi, D.A. Wellings, R. Lace, M.J. Horsburgh, R.L. Williams, A Novel Peptide Hydrogel for an Antimicrobial Bandage Contact Lens, Advanced healthcare materials 5(16) (2016) 2013-8.

[207] K. Choi, H.G. Park, Smart Reinvention of the Contact Lens with Graphene, ACS Nano 11(6) (2017) 5223-5226. 
[208] I.M. Carvalho, C.S. Marques, R.S. Oliveira, P.B. Coelho, P.C. Costa, D.C. Ferreira, Sustained drug release by contact lenses for glaucoma treatment-a review, J Control Release 202 (2015) 76-82.

[209] S. Holland, D. Morck, C. Schultz, Treatment of corneal defects with delayed reepithelization with a medical device/drug delivery system for epidermal growth factor, Clinical \& experimental ophthalmology 40(7) (2012) 662-668.

[210] C. Li, A. Chauhan, Ocular transport model for ophthalmic delivery of timolol through pHEMA contact lenses, Journal of drug delivery science and technology 17(1) (2007) 69-79.

[211] K.H. Hsu, B.E. Carbia, C. Plummer, A. Chauhan, Dual drug delivery from vitamin E loaded contact lenses for glaucoma therapy, Eur J Pharm Biopharm 94 (2015) 312-21.

[212] K.H. Hsu, P.L. de la Jara, A. Ariyavidana, J. Watling, B. Holden, Q. Garrett, A. Chauhan, Release of betaine and dexpanthenol from vitamin E modified silicone-hydrogel contact lenses, Curr Eye Res 40(3) (2015) 267-73.

[213] C.C. Peng, J. Kim, A. Chauhan, Extended delivery of hydrophilic drugs from siliconehydrogel contact lenses containing vitamin E diffusion barriers, Biomaterials 31(14) (2010) 403247.

[214] H.J. Jung, M. Abou-Jaoude, B.E. Carbia, C. Plummer, A. Chauhan, Glaucoma therapy by extended release of timolol from nanoparticle loaded silicone-hydrogel contact lenses, Journal of controlled release 165(1) (2013) 82-89.

[215] E. Garcia-Millan, S. Koprivnik, F.J. Otero-Espinar, Drug loading optimization and extended drug delivery of corticoids from pHEMA based soft contact lenses hydrogels via chemical and microstructural modifications, Int J Pharm 487(1-2) (2015) 260-9.

[216] G. Behl, J. Iqbal, N.J. O’Reilly, P. McLoughlin, L. Fitzhenry, Synthesis and characterization of poly (2-hydroxyethylmethacrylate) contact lenses containing chitosan nanoparticles as an ocular delivery system for dexamethasone sodium phosphate, Pharmaceutical research 33(7) (2016) 1638-1648.

[217] H.J. Jung, A. Chauhan, Temperature sensitive contact lenses for triggered ophthalmic drug delivery, Biomaterials 33(7) (2012) 2289-300.

[218] E.B. Souto, S. Doktorovova, E. Gonzalez-Mira, M.A. Egea, M.L. Garcia, Feasibility of lipid nanoparticles for ocular delivery of anti-inflammatory drugs, Curr Eye Res 35(7) (2010) 537-52.

[219] A. Danion, H. Brochu, Y. Martin, P. Vermette, Fabrication and characterization of contact lenses bearing surface-immobilized layers of intact liposomes, Journal of Biomedical Materials Research Part A 82(1) (2007) 41-51.

[220] H. Hiratani, A. Fujiwara, Y. Tamiya, Y. Mizutani, C. Alvarez-Lorenzo, Ocular release of timolol from molecularly imprinted soft contact lenses, Biomaterials 26(11) (2005) 1293-8.

[221] A. Tieppo, C.J. White, A.C. Paine, M.L. Voyles, M.K. McBride, M.E. Byrne, Sustained in vivo release from imprinted therapeutic contact lenses, J Control Release 157(3) (2012) 391-7.

[222] C.J. White, M.K. McBride, K.M. Pate, A. Tieppo, M.E. Byrne, Extended release of high molecular weight hydroxypropyl methylcellulose from molecularly imprinted, extended wear silicone hydrogel contact lenses, Biomaterials 32(24) (2011) 5698-705.

[223] L.C. Bengani, A. Chauhan, Extended delivery of an anionic drug by contact lens loaded with a cationic surfactant, Biomaterials 34(11) (2013) 2814-21.

[224] J.B. Ciolino, T.R. Hoare, N.G. Iwata, I. Behlau, C.H. Dohlman, R. Langer, D.S. Kohane, A drug-eluting contact lens, Investigative ophthalmology \& visual science 50(7) (2009) 3346-3352. 
[225] A. Carreira, P. Ferreira, M. Ribeiro, T. Correia, P. Coutinho, I. Correia, M. Gil, New drugeluting lenses to be applied as bandages after keratoprosthesis implantation, Int J Pharmaceut 477(1) (2014) 218-226.

[226] H. Yao, A.J. Shum, M. Cowan, I. Lahdesmaki, B.A. Parviz, A contact lens with embedded sensor for monitoring tear glucose level, Biosens Bioelectron 26(7) (2011) 3290-6.

[227] A.R. Lingley, M. Ali, Y. Liao, R. Mirjalili, M. Klonner, M. Sopanen, S. Suihkonen, T. Shen, B.P. Otis, H. Lipsanen, B.A. Parviz, A single-pixel wireless contact lens display. , Journal of Micromechanics and Microengineering 21(12) (2011) 125014.

[228] M. Rizwan, G.S. Peh, H.-P. Ang, N.C. Lwin, K. Adnan, J.S. Mehta, W.S. Tan, E.K. Yim, Sequentially-crosslinked bioactive hydrogels as nano-patterned substrates with customizable stiffness and degradation for corneal tissue engineering applications, Biomaterials 120 (2017) 139154.

[229] H. Yao, Y. Liao, A. Lingley, A. Afanasiev, I. Lähdesmäki, B. Otis, B. Parviz, A contact lens with integrated telecommunication circuit and sensors for wireless and continuous tear glucose monitoring, Journal of Micromechanics and Microengineering 22(7) (2012) 075007.

[230] G.Z. Chen, I.S. Chan, D.C. Lam, Capacitive contact lens sensor for continuous non-invasive intraocular pressure monitoring. , Sensors and Actuators A: Physical 203 (2013) 112-118.

[231] A.K. Geim, S.V. Dubonos, I.V. Grigorieva, K.S. Novoselov, A.A. Zhukov, S.Y. Shapoval, Microfabricated adhesive mimicking gecko foot-hair, Nature materials 2(7) (2003) 461-3.

[232] S. Majumdar, Q. Guo, M. Garza-Madrid, X. Calderon-Colon, D. Duan, P. Carbajal, O. Schein, M. Trexler, J. Elisseeff, Influence of collagen source on fibrillar architecture and properties of vitrified collagen membranes, Journal of Biomedical Materials Research Part B: Applied Biomaterials 104(2) (2016) 300-307.

[233] L. Du, X. Wu, Development and characterization of a full-thickness acellular porcine cornea matrix for tissue engineering, Artificial organs 35(7) (2011) 691-705.

[234] L. Du, X. Wu, K. Pang, Y. Yang, Histological evaluation and biomechanical characterisation of an acellular porcine cornea scaffold, Br J Ophthalmol 95(3) (2011) 410-4.

[235] M.A. Shafiq, R.A. Gemeinhart, B.Y. Yue, A.R. Djalilian, Decellularized human cornea for reconstructing the corneal epithelium and anterior stroma, Tissue Eng Part C Methods 18(5) (2012) 340-8.

[236] S. Sasaki, S. Funamoto, Y. Hashimoto, T. Kimura, T. Honda, S. Hattori, H. Kobayashi, A. Kishida, M. Mochizuki, In vivo evaluation of a novel scaffold for artificial corneas prepared by using ultrahigh hydrostatic pressure to decellularize porcine corneas, Molecular vision 15 (2009) 2022-8.

[237] S. Proulx, T. Bensaoula, O. Nada, C. Audet, J. d'Arc Uwamaliya, A. Devaux, G. Allaire, L. Germain, I. Brunette, Transplantation of a tissue-engineered corneal endothelium reconstructed on a devitalized carrier in the feline model, Invest Ophthalmol Vis Sci 50(6) (2009) 2686-94.

[238] X.C. Lin, Y.N. Hui, Y.S. Wang, H. Meng, Y.J. Zhang, Y. Jin, Lamellar keratoplasty with a graft of lyophilized acellular porcine corneal stroma in the rabbit, Veterinary ophthalmology 11(2) (2008) 61-6.

[239] Y. Hashimoto, S. Funamoto, S. Sasaki, T. Honda, S. Hattori, K. Nam, T. Kimura, M. Mochizuki, T. Fujisato, H. Kobayashi, A. Kishida, Preparation and characterization of decellularized cornea using high-hydrostatic pressurization for corneal tissue engineering, Biomaterials 31(14) (2010) 3941-8. 
[240] Y. Shao, Y. Yu, C.G. Pei, Q. Zhou, Q.P. Liu, G. Tan, J.M. Li, G.P. Gao, L. Yang, Evaluation of novel decellularizing corneal stroma for cornea tissue engineering applications, International journal of ophthalmology 5(4) (2012) 415-8.

[241] Y.J. Daoud, R. Smith, T. Smith, E.K. Akpek, D.E. Ward, W.J. Stark, The intraoperative impression and postoperative outcomes of gamma-irradiated corneas in corneal and glaucoma patch surgery, Cornea 30(12) (2011) 1387-91.

[242] A.P. Lynch, S.L. Wilson, M. Ahearne, Dextran Preserves Native Corneal Structure During Decellularization, Tissue Eng Part C Methods 22(6) (2016) 561-72.

[243] Z. Wu, L. Fan, B. Xu, Y. Lin, P. Zhang, X. Wei, Use of decellularized scaffolds combined with hyaluronic acid and basic fibroblast growth factor for skin tissue engineering, Tissue engineering. Part A 21(1-2) (2015) 390-402.

[244] Z. Su, H. Ma, Z. Wu, H. Zeng, Z. Li, Y. Wang, G. Liu, B. Xu, Y. Lin, P. Zhang, X. Wei, Enhancement of skin wound healing with decellularized scaffolds loaded with hyaluronic acid and epidermal growth factor, Mater Sci Eng C Mater Biol Appl 44 (2014) 440-8. 